\title{
On a Family of Triangular Arrays of Natural Numbers and the Tower of Hanoi with Four Pegs
}

\author{
Daniele Parisse \\ Airbus Defence and Space GmbH \\ Rechliner Str., 85077 Manching, Germany \\ daniele.parisse@t-online.de
}

June 14, 2021

\begin{abstract}
Several arrangements of the natural numbers $\mathbb{N}$ in triangular arrays $\left(T_{j}(n, k)\right)_{j \in \mathbb{N}}$, where $n \in \mathbb{N}$ denotes the row and $k \in \mathbb{N}$ the column, will be considered. These arrays are defined as follows: The first row has one element and each row has $j \in \mathbb{N}$ elements more than the preceding one, that is, the $n$th row has $j n-(j-1)$ elements. At first, a formula for the number appearing in the $n$th row and $k$ th column will be given and, secondly, we consider the inverse problem, i.e. given a number $N \in \mathbb{N}$ determine in which row $n$ and in which column $k$ one can find this number. Then some sequences appearing in these arrays together with the sequences of their partial sums will be studied. Finally, a surprising connection of $T_{1}(n, k)$ with an iterative minimal solution of the Tower of Hanoi with four pegs and $N \in \mathbb{N}$ discs will be shown. As a result it is possible to determine in advance how many subtowers with how many discs have to be built in the iterative minimal solution of the Tower of Hanoi with four pegs and $N \in \mathbb{N}$ discs.
\end{abstract}

Key words: $m$-dimensional figurate numbers, polygonal numbers, pyramidal numbers, figured numbers, Tower of Hanoi with four pegs

AMS subject classification (2000): 05A10, 05A15, 11B37

\section{Introduction}

Consider the sequence of the natural numbers arranged as follows in the triangular arrays $\left(T_{j}(n, k)\right)_{j \in \mathbb{N}}$, where $n \in \mathbb{N}$ denotes the row and $k \in \mathbb{N}$ the column: The first row has one element and each row has $j \in \mathbb{N}$ elements more than the preceding one, i.e. the $n$th row has $j n-(j-1)$ elements. This means that the number of elements in the array builds an arithmetic progression with difference $j$ and initial value 1 (see Tables 1, 2, and 3 for the cases $j=1,2,3$, where the last column gives the row sum sequence $\left.s_{j}^{(0)}(n)\right)$.

In this paper we intend to study these arrays and some special subsequences of these arrays together with their partial sums. The paper is organized as follows: First of all we

DOI: https://doi.org/10.5592/CO/CCD.2020.06 


\begin{tabular}{|r||r|r|r|r|r|r|r|r|r|r|r|r|r|r|}
\hline$n \backslash k$ & 1 & 2 & 3 & 4 & 5 & 6 & 7 & 8 & 9 & 10 & 11 & 12 & $\ldots$ & $s_{1}^{(0)}(n)$ \\
\hline \hline 1 & 1 & & & & & & & & & & & & & 1 \\
\hline 2 & 2 & 3 & & & & & & & & & & & & 5 \\
\hline 3 & 4 & 5 & 6 & & & & & & & & & & & 15 \\
\hline 4 & 7 & 8 & 9 & 10 & & & & & & & & & & 34 \\
\hline 5 & 11 & 12 & 13 & 14 & 15 & & & & & & & & & 65 \\
\hline 6 & 16 & 17 & 18 & 19 & 20 & 21 & & & & & & & & 111 \\
\hline 7 & 22 & 23 & 24 & 25 & 26 & 27 & 28 & & & & & & & 175 \\
\hline 8 & 29 & 30 & 31 & 32 & 33 & 34 & 35 & 36 & & & & & & 260 \\
\hline 9 & 37 & 38 & 39 & 40 & 41 & 42 & 43 & 44 & 45 & & & & & 369 \\
\hline 10 & 46 & 47 & 48 & 49 & 50 & 51 & 52 & 53 & 54 & 55 & & & & 505 \\
\hline 11 & 56 & 57 & 58 & 59 & 60 & 61 & 62 & 63 & 64 & 65 & 66 & & & 671 \\
\hline 12 & 67 & 68 & 69 & 70 & 71 & 72 & 73 & 74 & 75 & 76 & 77 & 78 & & 870 \\
\hline
\end{tabular}

Table 1: Triangular array $T_{1}(n, k), 1 \leq n \leq 12,1 \leq k \leq n$

\begin{tabular}{|r||r|r|r|r|r|r|r|r|r|r|r|r|r|r|r|}
\hline$n \backslash k$ & 1 & 2 & 3 & 4 & 5 & 6 & 7 & 8 & 9 & 10 & 11 & 12 & 13 & $\ldots$ & $s_{2}^{(0)}(n)$ \\
\hline \hline 1 & 1 & & & & & & & & & & & & & & 1 \\
\hline 2 & 2 & 3 & 4 & & & & & & & & & & & & 9 \\
\hline 3 & 5 & 6 & 7 & 8 & 9 & & & & & & & & & & 35 \\
\hline 4 & 10 & 11 & 12 & 13 & 14 & 15 & 16 & & & & & & & & 91 \\
\hline 5 & 17 & 18 & 19 & 20 & 21 & 22 & 23 & 24 & 25 & & & & & & 189 \\
\hline 6 & 26 & 27 & 28 & 29 & 30 & 31 & 32 & 33 & 34 & 35 & 36 & & & & 341 \\
\hline 7 & 37 & 38 & 39 & 40 & 41 & 42 & 43 & 44 & 45 & 46 & 47 & 48 & 49 & & 559 \\
\hline
\end{tabular}

Table 2: Triangular array $T_{2}(n, k), 1 \leq n \leq 7,1 \leq k \leq 2 n-1$

\begin{tabular}{|r||r|r|r|r|r|r|r|r|r|r|r|r|r|r|r|}
\hline$n \backslash k$ & 1 & 2 & 3 & 4 & 5 & 6 & 7 & 8 & 9 & 10 & 11 & 12 & 13 & $\ldots$ & $s_{3}^{(0)}(n)$ \\
\hline \hline 1 & 1 & & & & & & & & & & & & & & 1 \\
\hline 2 & 2 & 3 & 4 & 5 & & & & & & & & & & & 14 \\
\hline 3 & 6 & 7 & 8 & 9 & 10 & 11 & 12 & & & & & & & & 63 \\
\hline 4 & 13 & 14 & 15 & 16 & 17 & 18 & 19 & 20 & 21 & 22 & & & & & 175 \\
\hline 5 & 23 & 24 & 25 & 26 & 27 & 28 & 29 & 30 & 31 & 32 & 33 & 34 & 35 & & 377 \\
\hline
\end{tabular}

Table 3: Triangular array $T_{3}(n, k), 1 \leq n \leq 5,1 \leq k \leq 3 n-2$ 
define the triangular arrays and give a formula for the number appearing in the $n$th row and $k$ th column, then we consider the inverse problem, that is given a number $N \in \mathbb{N}$ we determine in which row $n$ and in which column $k$ one can find this number. After that we study four different sequences and their partial sums arising in these arrays and, finally, we will show a surprising connection of $T_{1}(n, k)$ with an iterative minimal solution of the Tower of Hanoi with four pegs and $N \in \mathbb{N}$ discs given by Hinz [8].

\section{Determination of $T_{j}(n, k)$}

We recall that the m-dimensional figurate numbers are defined as

$$
f_{m, j}(n):=\left(\begin{array}{c}
n+m-2 \\
m-1
\end{array}\right)+j\left(\begin{array}{c}
n+m-2 \\
m
\end{array}\right)=\frac{j n-(j-m)}{n+m-1}\left(\begin{array}{c}
n+m-1 \\
m
\end{array}\right)
$$

for all $m, j, n \in \mathbb{N}$, where $j$ is called the set number, $n$ the rank and $m$ the dimension of the figurate number. The number $s:=j+2 \geq 3$ is the number of sides of the geometric figure of $f_{m, j}(n)$ (cf. [1, pp. 185-199]).

Remark 2.1 The first few $m$-dimensional figurate numbers are for $j, n \in \mathbb{N}$

- $m=1: f_{1, j}(n)=\left(\begin{array}{c}n-1 \\ 0\end{array}\right)+j\left(\begin{array}{c}n-1 \\ 1\end{array}\right)=j n-(j-1)$.

These are called the linear group of figurate numbers. They are simply the terms of an arithmetic progression of difference $j \in \mathbb{N}$ starting with 1. Special cases of the linear group of figurate numbers are:

$j=1: f_{1,1}(n)=n$. These are the natural numbers, the sequence A000027 in the On-Line Encyclopedia of Integer Sequences (OEIS $\left.{ }^{\circledR}\right)$ [16].

$j=2: f_{1,2}(n)=2 n-1$. These are the odd numbers (or gnomic numbers) (A005408).

- $m=2: f_{2, j}(n)=\left(\begin{array}{l}n \\ 1\end{array}\right)+j\left(\begin{array}{l}n \\ 2\end{array}\right)=\frac{1}{2} n(j n-(j-2))$.

These are the plane figurate numbers (also called polygonal numbers or s-gonal numbers) $(s:=j+2 \geq 3)$. They are also denoted by $p_{s}^{n}$, that is $p_{s}^{n}=\frac{1}{2} n((s-$ $2) n-(s-4))$. Special cases of the polygonal numbers are:

$j=1: p_{3}^{n}=f_{2,1}(n)=\frac{1}{2} n(n+1)=\left(\begin{array}{c}n+1 \\ 2\end{array}\right)$. These are the triangular numbers (or 3-gonal numbers) (A000217), the sum of the first natural numbers from 1 to $n$.

$j=2: p_{4}^{n}=f_{2,2}(n)=n^{2}$. These are the square numbers (or 4-gonal numbers) (A000290), the sum of the first odd numbers from 1 to $2 n-1$.

$j=3: p_{5}^{n}=f_{2,3}(n)=\frac{1}{2} n(3 n-1)$. These are the pentagonal numbers (or 5 gonal numbers) $(\underline{\text { A000326 }})$. Note that $3 f_{2,3}(n)=\frac{1}{2} \cdot 3 n(3 n-1)=f_{2,1}(3 n-1)$ or $p_{5}^{n}=f_{2,3}(n)=\frac{1}{3} f_{2,1}(3 n-1)=\frac{1}{3} p_{3}^{3 n-1}$, that is every pentagonal number is one-third of some triangular number (cf. [4, p. 41]).

$j=4: p_{6}^{n}=f_{2,4}(n)=n(2 n-1)$. These are the hexagonal numbers (or 6-gonal numbers) (A000384). Note that this is a subsequence of $f_{2,1}(n)$, since $f_{2,4}(n)=$ $f_{2,1}(2 n-1)=f_{2,1}\left(f_{1,2}(n)\right)$, that is every hexagonal number is also a triangular number (cf. [4, p. 39]. 
- $m=3: f_{3, j}(n)=\left(\begin{array}{c}n+1 \\ 2\end{array}\right)+j\left(\begin{array}{c}n+1 \\ 3\end{array}\right)=\frac{1}{6} n(n+1)(j n-(j-3))$.

These are the solid figurate numbers (also called pyramidal numbers). They are also denoted by $P_{s}^{n}(s:=j+2 \geq 3)$, that is $P_{s}^{n}=\frac{1}{6} n(n+1)((s-2) n-(s-5))$. Special cases of the pyramidal numbers are:

$j=1: P_{3}^{n}=f_{3,1}(n)=\frac{1}{6} n(n+1)(n+2)=\left(\begin{array}{c}n+2 \\ 3\end{array}\right)$. These are the tetrahedral numbers $(\underline{\text { A000292) }}$.

$j=2: P_{4}^{n}=f_{3,2}(n)=\frac{1}{6} n(n+1)(2 n+1)$. These are the square pyramidal numbers (A000330), the sum of the first square numbers from 1 to $n^{2}$.

$j=3: P_{5}^{n}=f_{3,3}(n)=\frac{1}{2} n^{2}(n+1)$. These are the pentagonal pyramidal numbers (A002411).

$j=4: P_{6}^{n}=f_{3,4}(n)=\frac{1}{6} n(n+1)(4 n-1)$. These are the hexagonal pyramidal numbers $(\underline{A 002412)}$.

Finally, the numbers $f_{m, 1}(n)=\left(\begin{array}{c}n+m-2 \\ m-1\end{array}\right)+\left(\begin{array}{c}n+m-2 \\ m\end{array}\right)=\left(\begin{array}{c}n+m-1 \\ m\end{array}\right)=f_{m+1,0}(n)$ have also been called figured numbers with the alternative notation $\left\langle\begin{array}{c}n \\ m\end{array}\right\rangle$ (cf. [3, pp. 15-17]) or nth regular m-topic number with the notation $P_{n}^{(m)}$ (cf. [14, p. 211]). Note that we have used the recurrence relation for the binomial coefficients known as Pascal's rule or addition formula (cf. [7, Eq.(5.8), p.158]. With these notations we have $f_{m, j}(n)=$ $\left\langle\begin{array}{c}n \\ m-1\end{array}\right\rangle+j\left\langle\begin{array}{c}n-1 \\ m\end{array}\right\rangle$ and $f_{m, j}(n)=P_{n}^{(m-1)}+j P_{n-1}^{(m)}$, respectively. Combinatorially, there are $\left(\begin{array}{c}n+m-1 \\ m\end{array}\right)$ ways to choose $m$ elements from a set of $n$ elements $(m \leq n)$ where order does not count and repetitions are allowed. Note also that $f_{0,1}(n)=1$ for all $n \in \mathbb{N}$ and that by Eq.(2.1) the m-dimensional figurate numbers are generated by the figured numbers.

Theorem 2.2 Let $j, n, k \in \mathbb{N}$ and $k \in[j n-(j-1)]$, where $[p]:=\{1,2, \ldots, p\}$ for all natural numbers $p \geq 1$, then $T_{j}(n, k)$ is given by

$$
T_{j}(n, k)=\frac{n-1}{2}(j(n-1)-(j-2))+k=f_{2, j}(n)-f_{1, j}(n)+k=\left(\begin{array}{c}
n-1 \\
1
\end{array}\right)+j\left(\begin{array}{c}
n-1 \\
2
\end{array}\right)+k .
$$

Proof. The difference between two consecutive numbers in the $n$th row of $T_{j}(n, k)$ is by definition equal to 1 , that is

$$
\forall j, n, k \in \mathbb{N}, k \in[j n-j]: \quad T_{j}(n, k+1)=T_{j}(n, k)+1 .
$$

The solution of this arithmetic progression (in $k \in \mathbb{N}$ ) with difference 1 is given by

$$
T_{j}(n, k)=T_{j}(n, 1)+k-1, \quad k \in[j n-(j-1)],
$$

where $T_{j}(n, 1)$ is the initial value of the $n$th row. This value can be determined as follows: Since the $n$th row has $j n-(j-1)$ elements we obtain the recurrence relation in $n \geq 2$ $T_{j}(n, j n-(j-1)+1)=T_{j}(n-1, j n-(j-1)+1)+j n-(j-1)+1, \quad T_{j}(1,1)=1$, with the solution $T_{j}(n, j n-(j-1)+1)=\sum_{k=1}^{n}(j k-(j-1))=\frac{1}{2} j n(n+1)-(j-1) n=\frac{1}{2} n(j n-(j-2))=f_{2, j}(n)$ 
by using the formula for the sum of the first $n$ natural numbers $\sum_{k=1}^{n} k=\frac{1}{2} n(n+1)$. Hence,

$$
\begin{aligned}
T_{j}(n, 1) & =T_{j}(n-1, j(n-1)-(j-1))+1=\frac{1}{2}(n-1)(j(n-1)-(j-2))+1 \\
& =\frac{1}{2} n(j n-(j-2))-(j n-(j-1))+1=f_{2, j}(n)-f_{1, j}(n)+1 \\
& =f_{2, j}(n-1)=\left(\begin{array}{c}
n-1 \\
1
\end{array}\right)+j\left(\begin{array}{c}
n-1 \\
2
\end{array}\right) .
\end{aligned}
$$

Substituting this value into Eq.(2.3) we obtain Eq.(2.2).

The first three special cases of Eq.(2.2) are for all $n \geq 1$

$$
\begin{aligned}
& T_{1}(n, k)=\frac{1}{2} n(n-1)+k, \quad k \in[n], \\
& T_{2}(n, k)=(n-1)^{2}+k, \quad k \in[2 n-1] \\
& T_{3}(n, k)=\frac{1}{2}(n-1)(3 n-4)+k, \quad k \in[3 n-2] .
\end{aligned}
$$

For example $T_{3}(5,6)=\frac{1}{2} \cdot 4 \cdot(3 \cdot 5-4)+6=28$ as one can see in Table 3 .

Note that $f_{1, j}(n)$ gives the number of elements in the $n$th row of $T_{j}$ and $f_{2, j}(n)$ counts the total number of elements from the first row up to and including the $n$th row.

An immediate consequence of Theorem 2.2 is given in the following corollary.

Corollary 2.3 For all $j, n \in \mathbb{N}$ and for all $k \in[j n-(j-1)]$ :

$$
T_{j}(n, k)+T_{j}(n+2, k)=2 T_{j}(n+1, k)+j
$$

and

$$
T_{j}(n, k)+T_{j}(n+2, k+1)=2 T_{j}(n+1, k+1)+j-1
$$

Proof. For all $k \in[j n-(j-1)]$ we have by Eq. $(2.2): T_{j}(n, k)=\frac{1}{2}(n-1)(j(n-1)-(j-$ $2))+k$ and $T_{j}(n+2, k)=\frac{1}{2}(n+1)(j(n+1)-(j-2))+k$. Adding these two equations we obtain

$$
\begin{aligned}
T_{j}(n, k)+T_{j}(n+2, k) & =\frac{j}{2}\left((n-1)^{2}+(n+1)^{2}\right)-\frac{1}{2}(j-2)(n-1+n+1)+2 k \\
& =\frac{j}{2}\left(2 n^{2}+2\right)-(j-2) n+2 k \\
& =2 \cdot\left(\frac{n}{2} \cdot(j n-(j-2))+k\right)+j=2 T_{j}(n+1, k)+j .
\end{aligned}
$$

Similarly, using Eq.(2.4) we obtain $T_{j}(n, k)+T_{j}(n+2, k+1)=T_{j}(n, k)+T_{j}(n+2, k)+1=$ $2 T_{j}(n+1, k)+j+1=2 T_{j}(n+1, k+1)+j-1$.

Conversely, we now determine in which row and in which column a given $N \in \mathbb{N}$ can be found in $T_{j}(n, k)$. 
Theorem 2.4 Let $N \in \mathbb{N}$, then $N=T_{j}(n, k),(j, n, k \in \mathbb{N})$, where

$$
n=n_{j}(N)=\left\lfloor\frac{3 j-2+\sqrt{8 j(N-1)+(j-2)^{2}}}{2 j}\right\rfloor
$$

and

$$
k=k_{j}(N)=N-\frac{1}{2}\left(n_{j}(N)-1\right)\left(j\left(n_{j}(N)-1\right)-(j-2)\right) .
$$

Proof. $N$ is in the $n$th row of $T_{j}(n, k)$ if and only if

$$
T_{j}(n, 1) \leq N \leq T_{j}(n, j n-(j-1))=T_{j}(n+1,1)-1,
$$

or by $(2.2)$

$$
\frac{1}{2}(n-1)(j(n-1)-(j-2))+1 \leq N \leq \frac{1}{2} n(j(n+1)-2(j-1))+1-1,
$$

that is

$$
(n-1)(j n-2(j-1))+2 \leq 2 N \leq n(j n-(j-2))
$$

or, equivalently,

$$
j\left(n^{2}-\frac{3 j-2}{j} n+2\right) \leq 2 N \leq j\left(n^{2}-\frac{j-2}{j} n\right) .
$$

Dividing these inequalities by $j \neq 0$ and completing the square of the first and third inequality we obtain

$n^{2}-\frac{3 j-2}{j} n+2+\left(\frac{3 j-2}{2 j}\right)^{2}-\left(\frac{3 j-2}{2 j}\right)^{2} \leq \frac{2 N}{j} \leq n^{2}-\frac{j-2}{j} n+\left(\frac{j-2}{2 j}\right)^{2}-\left(\frac{j-2}{2 j}\right)^{2}$

or adding $\left(\frac{j-2}{2 j}\right)^{2}$ and simplifying

$$
\left(n-\frac{3 j-2}{2 j}\right)^{2}+\frac{2}{j} \leq \frac{2 N}{j}+\left(\frac{j-2}{2 j}\right)^{2} \leq\left(n-\frac{j-2}{2 j}\right)^{2} .
$$

Further, subtracting $\frac{2}{j}$ we get

$$
\left(n-\frac{3 j-2}{2 j}\right)^{2} \leq \frac{2(N-1)}{j}+\left(\frac{j-2}{2 j}\right)^{2} \leq\left(n-\frac{j-2}{2 j}\right)^{2}-\frac{2}{j}<\left(n-\frac{j-2}{2 j}\right)^{2}
$$

and these inequalities are equivalent to

$$
n-\frac{3 j-2}{2 j} \leq \frac{\sqrt{8 j(N-1)+(j-2)^{2}}}{2 j}<n-\frac{j-2}{2 j}
$$

or, finally,

$$
n \leq \frac{3 j-2+\sqrt{8 j(N-1)+(j-2)^{2}}}{2 j}<n+1 .
$$




\begin{tabular}{|r||r|r|r|r|r|r|r|r|r|r|r|r|r|r|r|r|}
\hline$N$ & 1 & 2 & 3 & 4 & 5 & 6 & 7 & 8 & 9 & 10 & 11 & 12 & 13 & 14 & 15 & 16 \\
\hline \hline$n_{1}(N)$ & 1 & 2 & 2 & 3 & 3 & 3 & 4 & 4 & 4 & 4 & 5 & 5 & 5 & 5 & 5 & 6 \\
\hline$n_{2}(N)$ & 1 & 2 & 2 & 2 & 3 & 3 & 3 & 3 & 3 & 4 & 4 & 4 & 4 & 4 & 4 & 4 \\
\hline$n_{3}(N)$ & 1 & 2 & 2 & 2 & 2 & 3 & 3 & 3 & 3 & 3 & 3 & 3 & 4 & 4 & 4 & 4 \\
\hline
\end{tabular}

Table $4: n_{j}(N), 1 \leq j \leq 3,1 \leq N \leq 16$

This is exactly the assertion (2.6) by the definition of the floor function (cf. [7, Eq.(3.5) (a), p. 69]). Finally, Eq.(2.7) follows from Eqs.(2.6) and (2.2).

For example, let $j=2$ and $N=19$, then by Eqs.(2.6) and (2.7) we obtain $n=$ $\lfloor 1+3 \sqrt{2}\rfloor=\lfloor 5.2426 \ldots\rfloor=5$ and $k=19-\frac{1}{2} \cdot 4 \cdot(2 \cdot(5-1)-0)=19-2 \cdot 8=3$ as one can see in Table 2 .

Remark 2.5 By Theorem 2.4 every number $N \in \mathbb{N}$ can be written as

$$
N=f_{2, j}\left(n_{j}(N)-1\right)+k_{j}(N),
$$

where $n_{j}(N)$ and $k_{j}(N)$ are given by Eqs.(2.6) and (2.7), respectively.

The family of sequences $\left(n_{j}(N)\right)_{N \in \mathbb{N}}$ with the parameter $j \in \mathbb{N}$ means "the number $N$ appears $f_{1, j}(N)=j N-(j-1)$ times".

The first three sequences are:

$$
\begin{array}{ll}
n_{1}(N)=\left\lfloor\frac{1}{2}(1+\sqrt{8 N-7})\right\rfloor, & (" N \text { appears } N \text { times" }) \quad(\underline{A 002024}), \\
n_{2}(N)=\lfloor 1+\sqrt{N-1}\rfloor, & (" N \text { appears } 2 N-1 \text { times" }) \quad(\underline{A 003059}), \\
n_{3}(N)=\left\lfloor\frac{1}{6}(7+\sqrt{24 N-23})\right\rfloor, \quad(" N \text { appears } 3 N-2 \text { times" }) \quad(\underline{A 083277}) .
\end{array}
$$

In Table 4 one can find the first few values of these sequences.

The family of sequences $\left(k_{j}(N)\right)_{N \in \mathbb{N}}$ with the parameter $j \in \mathbb{N}$ means "start counting the integers 1 to $j N-(j-1)$ followed by the integers 1 to $j N+1, N \in \mathbb{N}$, and so on".

The first three sequences are:

$k_{1}(N)=N-\frac{1}{2} n_{1}(N)\left(n_{1}(N)-1\right)$, ("Restart counting after each new integer") $(\underline{A 002260})$,

$k_{2}(N)=N-(\lfloor\sqrt{N-1}\rfloor)^{2}$, ("Restart counting after each new odd integer") $(\underline{A 071797})$,

$k_{3}(N)=N-\frac{1}{2}\left(n_{3}(N)-1\right)\left(3 n_{3}(N)-4\right),($ not available in the OEIS [16]).

In Table 5 one can find the first few values of these sequences.

Obviously, for $j \rightarrow \infty$, we obtain the sequences

$$
\left(n_{\infty}(N)\right)_{N \in \mathbb{N}}=(1,2,2,2,2 \ldots)=(2-(N=1))_{N \in \mathbb{N}},(\underline{A 040000})
$$




\begin{tabular}{|r||r|r|r|r|r|r|r|r|r|r|r|r|r|r|r|r|}
\hline$N$ & 1 & 2 & 3 & 4 & 5 & 6 & 7 & 8 & 9 & 10 & 11 & 12 & 13 & 14 & 15 & 16 \\
\hline \hline$k_{1}(N)$ & 1 & 1 & 2 & 1 & 2 & 3 & 1 & 2 & 3 & 4 & 1 & 2 & 3 & 4 & 5 & 1 \\
\hline$k_{2}(N)$ & 1 & 1 & 2 & 3 & 1 & 2 & 3 & 4 & 5 & 1 & 2 & 3 & 4 & 5 & 6 & 7 \\
\hline$k_{3}(N)$ & 1 & 1 & 2 & 3 & 4 & 1 & 2 & 3 & 4 & 5 & 6 & 7 & 1 & 2 & 3 & 4 \\
\hline
\end{tabular}

Table $5: k_{j}(N), 1 \leq j \leq 3,1 \leq N \leq 16$

\begin{tabular}{|r||c|c|c|c|r|r|r|r|r|r|r|r|r|r|r|r|}
\hline$N$ & 1 & 2 & 3 & 4 & 5 & 6 & 7 & 8 & 9 & 10 & 11 & 12 & 13 & 14 & 15 & 16 \\
\hline \hline$n_{1}^{(1)}(N)$ & 1 & 3 & 5 & 8 & 11 & 14 & 18 & 22 & 26 & 30 & 35 & 40 & 45 & 50 & 55 & 61 \\
\hline$n_{2}^{(1)}(N)$ & 1 & 3 & 5 & 7 & 10 & 13 & 16 & 19 & 22 & 26 & 30 & 34 & 38 & 42 & 46 & 50 \\
\hline$n_{3}^{(1)}(N)$ & 1 & 3 & 5 & 7 & 9 & 12 & 15 & 18 & 21 & 24 & 27 & 30 & 34 & 38 & 42 & 46 \\
\hline$k_{1}^{(1)}(N)$ & 1 & 2 & 4 & 5 & 7 & 10 & 11 & 13 & 16 & 20 & 21 & 23 & 26 & 30 & 35 & 36 \\
\hline$k_{2}^{(1)}(N)$ & 1 & 2 & 4 & 7 & 8 & 10 & 13 & 17 & 22 & 23 & 25 & 28 & 32 & 37 & 43 & 50 \\
\hline$k_{3}^{(1)}(N)$ & 1 & 2 & 4 & 7 & 11 & 12 & 14 & 17 & 21 & 26 & 32 & 39 & 40 & 42 & 45 & 49 \\
\hline
\end{tabular}

Table $6: n_{j}^{(1)}(N), k_{j}^{(1)}(N), 1 \leq j \leq 3,1 \leq N \leq 16$

and

$$
\left(k_{\infty}(N)\right)_{N \in \mathbb{N}}=(1,1,2,3,4 \ldots)=(N-(N>1))_{N \in \mathbb{N}},(\underline{A 028310}),
$$

where $(A)$ is Iverson's bracket convention, meaning 1 if the statement $A$ is true and 0 otherwise.

The sequences of the partial sums of these two families of sequences are by definition $n_{j}^{(1)}(N):=\sum_{i=1}^{N} n_{j}(i)$ and $k_{j}^{(1)}(N):=\sum_{i=1}^{N} k_{j}(i), N \in \mathbb{N}$.

In Table 6 one can find the first few values of the first three sequences of both families. The first sequence is ( $\underline{\mathrm{A} 060432})$, the second is $(\underline{\mathrm{A} 327672})$, the fourth is $(\underline{\mathrm{A} 014370})$, whereas the other three are not available in the OEIS [16].

Note that for $j \rightarrow \infty$ we obtain the sequences $n_{\infty}^{(1)}(N)=f_{1,2}(N)=2 N-1$ and $k_{\infty}^{(1)}(N)=T_{1}(N, 1)=\frac{1}{2} N(N-1)+1, N \in \mathbb{N}$.

\section{Four Particular Sequences}

In this section we shall study four special subsequences of the arrays $\left(T_{j}(n, k)\right)_{j \in \mathbb{N}}$ and the sequences of their partial sums.

The first sequence is obtained by choosing $k:=f_{1, j}(n)=j n-(j-1)$. We get

$$
d_{j}^{(0)}(n):=T_{j}(n, j n-(j-1))=f_{2, j}(n)=\frac{1}{2} n(j n-(j-2))
$$


and these are the polygonal numbers.

Choosing $k:=1$ we obtain by Theorem 2.2 the second sequence

$$
c_{j}^{(0)}(n):=T_{j}(n, 1)=f_{2, j}(n)-f_{1, j}(n)+1=n+j\left(\begin{array}{c}
n-1 \\
2
\end{array}\right)
$$

and this is the first column in Tables 1,2 and 3 .

Choosing $k:=n$ we obtain again by Theorem 2.2 the third sequence

$$
h_{j}^{(0)}(n):=T_{j}(n, n)=f_{2, j}(n)-f_{1, j}(n)+n=2 n-1+j\left(\begin{array}{c}
n-1 \\
2
\end{array}\right) .
$$

Notice that $h_{j}^{(0)}(n)=c_{j}^{(0)}(n)+n-1$.

Summing up the values in a row (see the last columns in Tables 1,2 and 3 for the cases $j \in[3])$ we get the fourth sequence

$$
s_{j}^{(0)}(n):=\sum_{k=1}^{j n-(j-1)} T_{j}(n, k) .
$$

In order to determine the partial sums of the first three sequences we need a lemma on figurate numbers.

Lemma 3.1 Let $j, m, n \in \mathbb{N}$, then

$$
\begin{gathered}
f_{m+1, j}(n)=\sum_{k=1}^{n} f_{m, j}(k) \\
(m+1) f_{m+1, j}(n)=(n+m-1) f_{m, j}(n)+f_{m, 1}(n)
\end{gathered}
$$

or, alternatively,

$$
(m+1) f_{m+1, j}(n)=n f_{m, j}(n+1)+(1-j) f_{m, 1}(n)
$$

and for $n \geq 2$

$$
\begin{gathered}
f_{m, j+1}(n)=f_{m, j}(n)+f_{m, 1}(n-1) \\
f_{m+1, j}(n)=f_{m, j}(n)+f_{m+1, j}(n-1) \\
f_{m, 2 j}(n)=f_{m, j}(n)+j f_{m, 1}(n-1) \\
f_{m, 2 j+1}(n)=f_{m, j}(n)+(j+1) f_{m, 1}(n-1) .
\end{gathered}
$$

Proof. By Eq.(2.1) and applying the formula of Chu-Shih-Chieh (1303), also called upper summation (cf. [7, Table 174, p.174]),

$$
\sum_{k=1}^{n}\left(\begin{array}{c}
k+m-1 \\
m
\end{array}\right)=\left(\begin{array}{c}
n+m \\
m+1
\end{array}\right)
$$


we obtain

$$
\begin{aligned}
\sum_{k=1}^{n} f_{m, j}(k) & =\sum_{k=1}^{n}\left(\left(\begin{array}{c}
k+m-2 \\
m-1
\end{array}\right)+j\left(\begin{array}{c}
k+m-2 \\
m
\end{array}\right)\right) \\
& =\sum_{k=1}^{n}\left(\begin{array}{c}
k+m-2 \\
m-1
\end{array}\right)+j \sum_{k=1}^{n}\left(\begin{array}{c}
k+m-2 \\
m
\end{array}\right) \\
& =\left(\begin{array}{c}
n+m-1 \\
m
\end{array}\right)+j\left(\begin{array}{c}
n+m-1 \\
m+1
\end{array}\right)=f_{m+1, j}(n)
\end{aligned}
$$

and this proves Eq.(3.5).

Again by Eq.(2.1) we have

$$
\begin{aligned}
(n+m-1) f_{m+1, j}(n) & =(n+m-1) \cdot\left(\left(\begin{array}{c}
n+m-2 \\
m-1
\end{array}\right)+j\left(\begin{array}{c}
n+m-2 \\
m
\end{array}\right)\right) \\
& =\frac{(n+m-1) !}{(m-1) !(n-1) !} \cdot \frac{m}{m}+j \cdot \frac{(n+m-1) !}{m !(n-2) !} \cdot \frac{m+1}{m+1} \\
& =\left(\begin{array}{c}
n+m-1 \\
m
\end{array}\right) m+j\left(\begin{array}{c}
n+m-1 \\
m+1
\end{array}\right)(m+1)
\end{aligned}
$$

and therefore

$$
(n+m-1) f_{m, j}(n)+f_{m, 1}(n)=(m+1) f_{m+1, j}(n),
$$

thus proving Eq.(3.6).

To prove the alternative Eq.(3.7) we note that for $n+1$ instead of $n$ we obtain from Eq.(2.1) $f_{m, j}(n+1)=\left(\begin{array}{c}n+m-1 \\ m-1\end{array}\right) m+j\left(\begin{array}{c}n+m-1 \\ m\end{array}\right)$, hence

$$
\begin{aligned}
(n+m-1) f_{m, j}(n) & =(n+m-1) \cdot\left(\left(\begin{array}{c}
n+m-2 \\
m-1
\end{array}\right)+j\left(\begin{array}{c}
n+m-2 \\
m
\end{array}\right)\right) \\
& =\frac{(n+m-1) !}{(m-1) !(n-1) !} \cdot \frac{n}{n}+j \cdot \frac{(n+m-1) !}{m !(n-2) !} \cdot \frac{n-1}{n-1} \\
& =n\left(\begin{array}{c}
n+m-1 \\
m-1
\end{array}\right)+(n-1) j\left(\begin{array}{c}
n+m-1 \\
m
\end{array}\right) \\
& =n f_{m, j}(n+1)-j f_{m, 1}(n) .
\end{aligned}
$$

Eq.(3.7) follows by inserting this equation into Eq.(3.6).

Now let $n \geq 2$, then by Eq.(2.1) we have

$$
\begin{aligned}
f_{m, j+1}(n)-f_{m, j}(n) & =\left(\begin{array}{c}
n+m-2 \\
m-1
\end{array}\right)+(j+1)\left(\begin{array}{c}
n+m-2 \\
m
\end{array}\right)-\left(\left(\begin{array}{c}
n+m-2 \\
m-1
\end{array}\right)+j\left(\begin{array}{c}
n+m-2 \\
m
\end{array}\right)\right) \\
& =\left(\begin{array}{c}
n+m-2 \\
m
\end{array}\right)=\left(\begin{array}{c}
n-1)+m-1 \\
m
\end{array}\right)=f_{m, 1}(n-1)
\end{aligned}
$$

and this proves Eq.(3.8).

Again by Eq.(2.1) it follows

$$
\begin{aligned}
f_{m+1, j}(n)-f_{m, j}(n) & =\left(\begin{array}{c}
n+m-1 \\
m
\end{array}\right)+j\left(\begin{array}{c}
n+m-1 \\
m+1
\end{array}\right)-\left(\left(\begin{array}{c}
n+m-2 \\
m-1
\end{array}\right)+j\left(\begin{array}{c}
n+m-2 \\
m
\end{array}\right)\right) \\
& =\left(\begin{array}{c}
n+m-1 \\
m
\end{array}\right)-\left(\begin{array}{c}
n+m-2 \\
m-1
\end{array}\right)+j\left(\left(\begin{array}{c}
n+m-1 \\
m+1
\end{array}\right)-\left(\begin{array}{c}
n+m-2 \\
m
\end{array}\right)\right) \\
& =\left(\begin{array}{c}
n+m-2 \\
m
\end{array}\right)+j\left(\begin{array}{c}
n+m-2 \\
m+1
\end{array}\right)=f_{m+1, j}(n-1)
\end{aligned}
$$


by using repeatedly Pascal's rule and this proves Eq.(3.9).

By definition we have $f_{m, 2 j}(n)=\left(\begin{array}{c}n+m-2 \\ m-1\end{array}\right)+2 j\left(\begin{array}{c}n+m-2 \\ m\end{array}\right)$ and $f_{m, j}(n)+j f_{m, 1}(n-1)=$ $\left(\begin{array}{c}n+m-2 \\ m-1\end{array}\right)+j\left(\begin{array}{c}n+m-2 \\ m\end{array}\right)+j\left(\begin{array}{c}n+m-2 \\ m\end{array}\right)=\left(\begin{array}{c}n+m-2 \\ m-1\end{array}\right)+2 j\left(\begin{array}{c}n+m-2 \\ m\end{array}\right)$ and this proves Eq. (3.10).

Finally, again by definition and applying Pascal's rule we have $f_{m, 2 j+1}(n)=\left(\begin{array}{c}n+m-2 \\ m-1\end{array}\right)+$ $(2 j+1)\left(\begin{array}{c}n+m-2 \\ m\end{array}\right)=\left(\begin{array}{c}n+m-1 \\ m\end{array}\right)+2 j\left(\begin{array}{c}n+m-2 \\ m\end{array}\right)$ and $f_{m, j}(n)+(j+1) f_{m, 1}(n-1)=\left(\begin{array}{c}n+m-2 \\ m-1\end{array}\right)+$ $j\left(\begin{array}{c}n+m-2 \\ m\end{array}\right)+(j+1)\left(\begin{array}{c}n+m-2 \\ m\end{array}\right)=\left(\begin{array}{c}n+m-1 \\ m\end{array}\right)+2 j\left(\begin{array}{c}n+m-2 \\ m\end{array}\right)$. This proves Eq.(3.11) and therefore the lemma is proved.

We now make some remarks to Lemma 3.1.

Remark 3.2 Formula (3.5) shows that for $m \geq 1$ the $(m+1)$-dimensional figurate numbers are the partial sums of the $m$-dimensional figurate numbers. In particular, for $m=2$ and $s=j+2 \geq 3$, we obtain

$$
P_{s}^{n}=\sum_{k=1}^{n} p_{s}^{k} .
$$

Remark 3.3 For $m=1$ we obtain from Eq.(3.6)

$$
f_{2, j}(n)=\frac{1}{2}\left(n f_{1, j}(n)+f_{1,1}(n)\right)=\frac{1}{2} n\left(f_{1, j}(n)+1\right) .
$$

This formula is a generalization of the sum of the first $n$ natural numbers. (Choose in $E q$.(3.14) $j=1$, then $f_{1,1}(n)=n$ and by Eq.(3.5) $f_{2,1}(n)=\sum_{k=1}^{n} f_{1,1}(k)=\sum_{k=1}^{n} k$, hence $\sum_{k=1}^{n} k=\frac{1}{2} n(n+1)$.)

For $m=2$ we get the formula of the Roman geometers and pupils of Heron of Alexandria Epaphroditus and Vitrius Rufus (about 100 A.D.), since $f_{3, j}(n)=\frac{1}{3}\left((n+1) f_{2, j}(n)+\right.$ $\left.f_{2,1}(n)\right)=\frac{1}{6}(n+1)\left(2 f_{2, j}(n)+n\right)$ or, equivalently, for $s \geq 3$

$$
P_{s}^{n}=\frac{1}{6}(n+1)\left(2 p_{s}^{n}+n\right),
$$

which shows how to obtain a pyramidal number $P_{s}^{n}$ from the corresponding polygonal number $p_{s}^{n}$. Note also that Eq.(3.15) is a generalization of the sum of the first $n$ square numbers. (Choose in Eq.(3.15) $j=2$, then $f_{2,2}(n)=n^{2}$ and by Eq.(3.5) $f_{3,2}(n)=$ $\sum_{k=1}^{n} f_{2,2}(k)=\sum_{k=1}^{n} k^{2}$, thus obtaining $\sum_{k=1}^{n} k^{2}=\frac{1}{6} n(n+1)(2 n+1)$.)

Moreover, the formula (3.6) shows that for all $j, n \in \mathbb{N}$ the $m$-dimensional figurate numbers $f_{m, j}(n)$ are the solution of the recurrence relation in $m \in \mathbb{N}$

$$
a_{m+1, j}(n)=\frac{n+m-1}{m+1} \cdot a_{m, j}(n)+\frac{1}{m+1}\left(\begin{array}{c}
n+m-1 \\
m
\end{array}\right)
$$

with the initial value $a_{1, j}(n)=j n-(j-1)$.

Remark 3.4 For $j=1$ Eq.(3.7) gives $(m+1) f_{m+1,1}(n)=n f_{m, 1}(n+1)$ (P.de Fermat(1636)), since $(m+1)\left(\begin{array}{c}n+m \\ m+1\end{array}\right)=\frac{(m+1)(n+m) !}{(m+1) !(n-1) !}=\frac{n(n+m) !}{m ! n !}=n\left(\begin{array}{c}n+m \\ m\end{array}\right)=n f_{m, 1}(n+1)$. 
Remark 3.5 The special cases of Eq.(3.8) for $m=2: p_{s+1}^{n}=p_{s}^{n}+p_{3}^{n-1}$ and for $m=3: P_{s+1}^{n}=P_{s}^{n}+P_{3}^{n-1}(n \geq 2$ and $s \geq 3)$ have been given by Nicomachus of Gerasa (about 100 A.D.). Note that from these formulae one can obtain by induction $p_{s}^{n}=p_{3}^{n}+(s-3) p_{3}^{n-1}$ (C.G. Bachet (1621)) and $P_{s}^{n}=P_{3}^{n}+(s-3) P_{3}^{n-1}$ (F. Maurolico (1575)) $(n \geq 2$ and $s \geq 3)$.

Remark 3.6 For $m=2$ and $j=2$ Eq.(3.9) gives $p_{6}^{n}=f_{2,4}(n)=2 f_{2,1}(n-1)+$ $f_{2,2}(n)=2 p_{3}^{n-1}+n^{2}$, (F. Maurolico (1575)), whereas for $m=2$ and $j=1$ Eq.(3.10) gives $p_{5}^{n}=f_{2,3}(n)=2 f_{2,1}(n-1)+f_{2,1}(n)=2 p_{3}^{n-1}+p_{3}^{n}$. Since $p_{3}^{n}=p_{3}^{n-1}+n$ we obtain in this way $p_{5}^{n}=3 p_{3}^{n-1}+n$ (Nicomachus of Gerasa (about 100 A.D.)). In general, we have for $n \geq 2$ and $s \geq 3: p_{s}^{n}=p_{3}^{n}+(s-3) p_{3}^{n-1}=p_{3}^{n-1}+n+(s-3) p_{3}^{n-1}=(s-2) p_{3}^{n-1}+n$ and $p_{s}^{n}=p_{3}^{n}+(s-3) p_{3}^{n-1}=p_{3}^{n}+p_{3}^{n-1}+(s-4) p_{3}^{n-1}=(s-4) p_{3}^{n-1}+n^{2}$, since $p_{3}^{n}+p_{3}^{n-1}=\left(\begin{array}{c}n+1 \\ 2\end{array}\right)+\left(\begin{array}{c}n \\ 2\end{array}\right)=n^{2}$.

Before proceeding, we recall that the hypersequence of the mth generation, $m \in \mathbb{N}$, of an arbitrary sequence $\left(a_{n}\right)_{n \in \mathbb{N}}$ is defined as $a_{n}^{(m)}:=\sum_{k=1}^{n} a_{k}^{(m-1)}, a_{n}^{(0)}:=a_{n}$.

Proposition 3.7 Let $m \geq 1$ and $d_{j}^{(m)}(n):=\sum_{k=1}^{n} d_{j}^{(m-1)}(k), c_{j}^{(m)}(n):=\sum_{k=1}^{n} c_{j}^{(m-1)}(k)$, $h_{j}^{(m)}(n):=\sum_{k=1}^{n} h_{j}^{(m-1)}(k)$ be the hypersequences of the mth generation of $\left(d_{j}^{(0)}(n)\right)_{n \in \mathbb{N}}$, $\left(c_{j}^{(0)}(n)\right)_{n \in \mathbb{N}}$ and $\left(h_{j}^{(0)}(n)\right)_{n \in \mathbb{N}}$, respectively. Then for all $m \in \mathbb{N}_{0}$ and all $n, j \in \mathbb{N}$ :

$$
\begin{gathered}
d_{j}^{(m)}(n)=f_{m+2, j}(n)=\left(\begin{array}{c}
n+m \\
m+1
\end{array}\right)+j\left(\begin{array}{c}
n+m \\
m+2
\end{array}\right) \\
c_{j}^{(m)}(n)=f_{m+1,1}(n)+j f_{m+2,1}(n-2)=\left(\begin{array}{c}
n+m \\
m+1
\end{array}\right)+j\left(\begin{array}{c}
n+m-1 \\
m+2
\end{array}\right) \\
h_{j}^{(m)}(n)=f_{m+1,2}(n)+j f_{m+2,1}(n-2)=\left(\begin{array}{c}
n+m+1 \\
m+2
\end{array}\right)+(j-1)\left(\begin{array}{c}
n+m-1 \\
m+2
\end{array}\right) .
\end{gathered}
$$

Proof. By definition (3.1) $d_{j}^{(0)}(n)=f_{2, j}(n)$ and, therefore, applying Eq.(3.5) $m$ times we obtain the first equation. The second one follows immediately from Eq.(2.1) and this proves (3.17).

Next, we obtain by Eq.(3.2) $c_{j}^{(0)}(n)=f_{1,1}(n)+j f_{2,1}(n-2)$, since $f_{1,1}(n)=n$ and $f_{2,1}(n)=\left(\begin{array}{c}n+1 \\ 2\end{array}\right)$. Hence, applying Eq.(3.5) $m$ times we get $c_{j}^{(m)}(n)=f_{m+1,1}(n)+$ $j f_{m+2,1}(n-2)$. The second equation now follows from Eq.(2.1) and this proves (3.18).

Finally, since $f_{1,2}(n)=2 n-1$ we have by $(3.3) h_{j}^{(0)}(n)=f_{1,2}(n)+j f_{2,1}(n-2)$. Applying Eq.(3.5) $m$ times we obtain $h_{j}^{(m)}(n)=f_{m+1,2}(n)+j f_{m+2,1}(n-2)$ and this proves the first equation of (3.19). The second equation follows from (2.1) by noting that

$$
\begin{aligned}
f_{m+1,2}(n) & =\left(\begin{array}{c}
n+m-1 \\
m
\end{array}\right)+2\left(\begin{array}{c}
n+m-1 \\
m+1
\end{array}\right) \\
& =\left(\begin{array}{c}
n+m \\
m+1
\end{array}\right)+\left(\begin{array}{c}
n+m-1 \\
m+1
\end{array}\right)+\left(\begin{array}{c}
n+m-1 \\
m+2
\end{array}\right)-\left(\begin{array}{c}
n+m-1 \\
m+2
\end{array}\right) \\
& =\left(\begin{array}{c}
n+m \\
m+1
\end{array}\right)+\left(\begin{array}{c}
n+m \\
m+2
\end{array}\right)-\left(\begin{array}{c}
n+m-1 \\
m+2
\end{array}\right)=\left(\begin{array}{c}
n+m+1 \\
m+2
\end{array}\right)-\left(\begin{array}{c}
n+m-1 \\
m+2
\end{array}\right)
\end{aligned}
$$


and using Pascal's rule several times.

We notice that $h_{j}^{(m)}(n)=c_{j}^{(m)}(n)+f_{m+1,1}(n)-f_{m, 1}(n)=c_{j}^{(m)}(n)+\left(\begin{array}{c}n+m+1 \\ m+1\end{array}\right)$ by Pascal's rule.

Special cases of $c_{j}^{(m)}(n)$ are

- $m=0: c_{j}^{(0)}(n)=\left(\begin{array}{c}n \\ 1\end{array}\right)+j\left(\begin{array}{c}n-1 \\ 2\end{array}\right)$.

$j=1: c_{1}^{(0)}(n)=\left(\begin{array}{c}n \\ 1\end{array}\right)+\left(\begin{array}{c}n-1 \\ 2\end{array}\right)=\left(\begin{array}{l}n \\ 2\end{array}\right)+1$. These are the centered polygonal numbers ( $\underline{\mathrm{A} 000124})$. They give the maximal number of regions into which $n \in \mathbb{N}$ lines separate the plane (cf. [15, pp. 124-126]).

$j=2: c_{2}^{(0)}(n)=n+2\left(\begin{array}{c}n-1 \\ 2\end{array}\right)=n^{2}-2 n+2=(n-1)^{2}+1(\underline{\mathrm{A} 002522})$. Note that $c_{2}^{(0)}(n)$ is one less than the arithmetic mean of its neighbours.

$j=3: c_{3}^{(0)}(n)=n+3\left(\begin{array}{c}n-1 \\ 2\end{array}\right)=\frac{1}{2}\left(3 n^{2}-7 n+6\right)(\underline{\text { A1436899 }})$.

- $m=1: c_{j}^{(1)}(n)=\left(\begin{array}{c}n+1 \\ 2\end{array}\right)+j\left(\begin{array}{l}n \\ 3\end{array}\right)$.

$j=1: c_{1}^{(1)}(n)=\frac{1}{6} n\left(n^{2}+5\right)=\left(\begin{array}{l}n \\ 1\end{array}\right)+\left(\begin{array}{l}n \\ 2\end{array}\right)+\left(\begin{array}{l}n \\ 3\end{array}\right)$. These numbers are the 3-dimensional analog of centered polygonal numbers ( $\underline{\mathrm{A} 004006})$.

$j=2: c_{2}^{(1)}(n)=\frac{1}{6} n\left(2 n^{2}-3 n+7\right)=\left(\begin{array}{l}n \\ 1\end{array}\right)+\left(\begin{array}{l}n \\ 2\end{array}\right)+2\left(\begin{array}{l}n \\ 3\end{array}\right)(\underline{\mathrm{A} 081489})$.

$j=3: c_{3}^{(1)}(n)=\frac{1}{2} n\left(n^{2}-2 n+3\right)=\left(\begin{array}{l}n \\ 1\end{array}\right)+2\left(\begin{array}{l}n \\ 2\end{array}\right)+3\left(\begin{array}{l}n \\ 3\end{array}\right)(\underline{\mathrm{A} 064808})$. This is the sequence of the $n$th $(n+1)$-gonal number.

- $m=2: c_{j}^{(2)}(n)=\left(\begin{array}{c}n+2 \\ 3\end{array}\right)+j\left(\begin{array}{c}n+1 \\ 4\end{array}\right)$.

$j=1: c_{1}^{(2)}(n)=\frac{1}{24} n(n+1)\left(n^{2}+n+10\right)(\underline{\mathrm{A} 006522})$.

$j=2: c_{2}^{(2)}(n)=\frac{1}{12} n(n+1)\left(n^{2}-n+6\right)$. This sequence is not available in the OEIS [16].

$j=3: c_{3}^{(2)}(n)=\frac{1}{24} n(n+1)\left(3 n^{2}-5 n+14\right)$. This sequence is not available in the OEIS [16].

and special cases of $h_{j}^{(m)}(n)$ are

- $m=0: h_{j}^{(0)}(n)=\left(\begin{array}{c}n+1 \\ 2\end{array}\right)+(j-1)\left(\begin{array}{c}n-1 \\ 2\end{array}\right)=2 n-1+j\left(\begin{array}{c}n-1 \\ 2\end{array}\right)$.

$j=1: h_{1}^{(0)}(n)=\left(\begin{array}{c}n+1 \\ 2\end{array}\right)$. These are the triangular numbers ( $\left.\underline{\mathrm{A} 000217}\right)$.

$j=2: h_{2}^{(0)}(n)=n^{2}-n+1$. These are the Hogben's centered polygonal numbers $(\underline{\mathrm{A} 002061})$.

$j=3: h_{3}^{(0)}(n)=\frac{1}{2}\left(3 n^{2}-5 n+4\right)(\underline{\mathrm{A} 104249})$.

- $m=1: h_{j}^{(1)}(n)=\left(\begin{array}{c}n+2 \\ 3\end{array}\right)+(j-1)\left(\begin{array}{l}n \\ 3\end{array}\right)=n^{2}+j\left(\begin{array}{l}n \\ 3\end{array}\right)$.

$j=1: h_{1}^{(1)}(n)=\left(\begin{array}{c}n+2 \\ 3\end{array}\right)$. These are the tetrahedral numbers ( $\left.\underline{\mathrm{A} 000292}\right)$.

$j=2: h_{2}^{(1)}(n)=\frac{1}{3} n\left(n^{2}+2\right)(\underline{\mathrm{A} 006527})$.

$j=3: h_{3}^{(1)}(n)=\frac{1}{2} n\left(n^{2}-n+2\right)(\underline{\mathrm{A} 006000})$. 
- $m=2: h_{j}^{(2)}(n)=\left(\begin{array}{c}n+3 \\ 4\end{array}\right)+(j-1)\left(\begin{array}{c}n+1 \\ 4\end{array}\right)=\frac{1}{6} n(n+1)(2 n+1)+j\left(\begin{array}{c}n+1 \\ 4\end{array}\right)$.

$j=1: h_{1}^{(2)}(n)=\left(\begin{array}{c}n+3 \\ 4\end{array}\right)$. These are the pentatope numbers $(\underline{\mathrm{A} 000332})$.

$j=2: h_{2}^{(2)}(n)=\frac{1}{12} n(n+1)\left(n^{2}+n+4\right)(\underline{\mathrm{A} 006007})$.

$j=3: h_{3}^{(2)}(n)=\frac{1}{24} n(n+1)\left(3 n^{2}-n+10\right)(\underline{\mathrm{A} 133252})$

In order to determine the hypersequence of the $m$ th generation of the fourth sequence (3.4) we need a general formula which gives the hypersequence of the $m$ th generation of an arbitrary sequence as the partial sums of another sequence.

Theorem 3.8 Let $\left(a_{n}\right)_{n \in \mathbb{N}}$ be an arbitrary sequence (of real numbers) and $a_{n}^{(m)}:=$ $\sum_{k=1}^{n} a_{k}^{(m-1)}, m \in \mathbb{N}, a_{n}^{(0)}:=a_{n}$, be the hypersequence of the mth generation of $\left(a_{n}\right)_{n \in \mathbb{N}}$. Then for all $m \in \mathbb{N}_{0}$ :

$$
a_{n}^{(m)}=\sum_{k=1}^{n}\left(\begin{array}{c}
n+m-1-k \\
m-1
\end{array}\right) a_{k}=\sum_{k=1}^{n}\left(\begin{array}{c}
m+k-2 \\
m-1
\end{array}\right) a_{n-k+1} .
$$

Proof. By induction on $m \geq 0$. For $m=0$ we have $\left(\begin{array}{c}n-1-k \\ -1\end{array}\right)=0$ for $k \in[n-1]$ and for $k=n$ it is $\left(\begin{array}{l}-1 \\ -1\end{array}\right)=1$, hence $a_{n}^{(0)}=a_{n}$.

For $m=1$ we have $\left(\begin{array}{c}n+1-1-k \\ 0\end{array}\right)=1$ for all $k \in[n]$, hence $a_{n}^{(1)}:=\sum_{k=1}^{n} a_{k}$.

Let us now assume that the formula is true for some $m \geq 0$, then

$$
\begin{aligned}
& a_{n}^{(m+1)}=\sum_{k=1}^{n} a_{k}^{(m)}=\sum_{k=1}^{n}\left(\sum_{i=1}^{k}\left(\begin{array}{c}
k+m-1-i \\
m-1
\end{array}\right) a_{i}\right) \\
& =\left(\begin{array}{c}
m-1 \\
m-1
\end{array}\right) a_{1}+\left[\left(\begin{array}{c}
2+m-1-1 \\
m-1
\end{array}\right) a_{1}+\left(\begin{array}{c}
2+m-1-2 \\
m-1
\end{array}\right) a_{2}\right]+ \\
& +\left[\left(\begin{array}{c}
3+m-1-1 \\
m-1
\end{array}\right) a_{1}+\left(\begin{array}{c}
3+m-1-2 \\
m-1
\end{array}\right) a_{2}+\left(\begin{array}{c}
3+m-1-3 \\
m-1
\end{array}\right) a_{3}\right]+\ldots \\
& \ldots+\left[\left(\begin{array}{c}
n+m-1-1 \\
m-1
\end{array}\right) a_{1}+\left(\begin{array}{c}
n+m-1-2 \\
m-1
\end{array}\right) a_{2}+\cdots+\left(\begin{array}{c}
n+m-1-n \\
m-1
\end{array}\right) a_{n}\right] .
\end{aligned}
$$

Collecting the coefficients of $a_{1}, a_{2}, \ldots, a_{n}$ we obtain

$$
\begin{aligned}
a_{n}^{(m+1)} & =\left[\left(\begin{array}{c}
m-1 \\
m-1
\end{array}\right)+\left(\begin{array}{c}
m \\
m-1
\end{array}\right)+\left(\begin{array}{c}
m+1 \\
m-1
\end{array}\right)+\cdots+\left(\begin{array}{c}
n+m-2 \\
m-1
\end{array}\right)\right] a_{1}+ \\
& +\left[\left(\begin{array}{c}
m-1 \\
m-1
\end{array}\right)+\left(\begin{array}{c}
m \\
m-1
\end{array}\right)+\left(\begin{array}{c}
m+1 \\
m-1
\end{array}\right)+\ldots+\left(\begin{array}{c}
n+m-3 \\
m-1
\end{array}\right)\right] a_{2}+\ldots+\left(\begin{array}{c}
m-1 \\
m-1
\end{array}\right) a_{n}
\end{aligned}
$$

Making use of the formula (3.12) for $m-1$ instead of $m$ and noting that $\left(\begin{array}{c}m-1 \\ m-1\end{array}\right)=\left(\begin{array}{c}m \\ m\end{array}\right)$ we finally obtain

$$
\begin{aligned}
a_{n}^{(m+1)} & =\left(\begin{array}{c}
n+m-1 \\
m
\end{array}\right) a_{1}+\left(\begin{array}{c}
n+m-2 \\
m
\end{array}\right) a_{2}+\cdots+\left(\begin{array}{c}
m \\
m
\end{array}\right) a_{n} \\
& =\sum_{k=1}^{n}\left(\begin{array}{c}
n+m-k \\
m
\end{array}\right) a_{k}=\sum_{k=1}^{n}\left(\begin{array}{c}
n+(m+1)-1-k \\
(m+1)-1
\end{array}\right) a_{k}
\end{aligned}
$$

and this verifies Eq.(3.20) for $m+1$ and in conclusion we have proved the theorem for all $m \in \mathbb{N}_{0}$. The second equation follows from the fact that $k \in[n]$ if and only if $n-k+1 \in[n]$. 
Remark 3.9 Formula (3.20) seems to be the discrete analogon of the formula of Cauchy

$$
\int_{x_{0}}^{x} \int_{x_{0}}^{x_{1}} \cdots \int_{x_{0}}^{x_{n-1}} f\left(x_{n}\right) d x_{n} \cdots d x_{2} d x_{1}=\frac{1}{(n-1) !} \int_{x_{0}}^{x} f(t)(x-t)^{n-1} d t
$$

according to which an $n$-fold repeated integral of the function $f(t)$ over the interval $\left[x_{0}, x\right]$ is expressed by a single integral with the integrand $\frac{(x-t)^{n-1}}{(n-1) !} f(t)$ over the same interval. Note that formula (3.21) says that $y(x):=\frac{1}{(n-1) !} \int_{x_{0}}^{x} f(t)(x-t)^{n-1} d t$ is the solution of the $n$th order differential equation $y^{(n)}(x)=f(x)$ with the initial values $y\left(x_{0}\right)=y^{\prime}\left(x_{0}\right)=$ $\cdots=y^{(n-1)}\left(x_{0}\right)=0$.

Before applying Theorem (3.8) to the sequences $(1)_{n \in \mathbb{N}},(n)_{n \in \mathbb{N}},\left(n^{2}\right)_{n \in \mathbb{N}}$ and $\left(n^{3}\right)_{n \in \mathbb{N}}$ we need the following lemma.

Lemma 3.10 For all $k, m, n \in \mathbb{N}$ :

$$
f_{m+k, k}(n)=k f_{m+k, 1}(n)-(k-1) f_{m+k-1,1}(n)
$$

Proof. Since $f_{m, 1}(n)=\left(\begin{array}{c}n+m-1 \\ m\end{array}\right)$ we obtain by Pascal's rule

$$
k f_{m+k, 1}(n)=k\left(\begin{array}{c}
n+m+k-1 \\
m+k
\end{array}\right)=k\left(\left(\begin{array}{c}
n+m+k-2 \\
m+k
\end{array}\right)+\left(\begin{array}{c}
n+m+k-2 \\
m+k-1
\end{array}\right)\right)
$$

and

$$
(k-1) f_{m+k, 1}(n)=(k-1)\left(\begin{array}{c}
n+m+k-2 \\
m+k-1
\end{array}\right) .
$$

Hence,

$$
\begin{aligned}
k f_{m+k, 1}(n)-(k-1) f_{m+k-1,1}(n) & =k\left(\left(\begin{array}{c}
n+m+k-2 \\
m+k
\end{array}\right)+\left(\begin{array}{c}
n+m+k-2 \\
m+k-1
\end{array}\right)\right)-(k-1)\left(\begin{array}{c}
n+m+k-2 \\
m+k-1
\end{array}\right) \\
& =\left(\begin{array}{c}
n+m+k-2 \\
m+k-1
\end{array}\right)+k\left(\begin{array}{c}
n+m+k-2 \\
m+k
\end{array}\right)
\end{aligned}
$$

and the last term on the right-hand side is by definition $f_{m+k, k}(n)$.

Alternatively, for $m-2$ instead of $m$, we obtain for all $k, m, n \in \mathbb{N}$

$$
f_{m+k-2, k}(n)=k f_{m+k-2,1}(n)-(k-1) f_{m+k-3,1}(n) .
$$

In particular, for $k=3$ and $m=1, p_{5}^{n}=f_{2,3}(n)=3 f_{2,1}(n)-2 f_{1,1}(n)=3 p_{3}^{n}-2 n$.

Lemma 3.11 For all $m, n \in \mathbb{N}$ :

$$
\begin{aligned}
& \sum_{k=1}^{n}\left(\begin{array}{c}
n+m-1-k \\
m-1
\end{array}\right)=\left(\begin{array}{c}
n+m-1 \\
m
\end{array}\right)=f_{m, 1}(n) \\
& \sum_{k=1}^{n} k\left(\begin{array}{c}
n+m-1-k \\
m-1
\end{array}\right)=\left(\begin{array}{c}
n+m \\
m+1
\end{array}\right)=f_{m+1,1}(n)
\end{aligned}
$$




$$
\begin{gathered}
\sum_{k=1}^{n} k^{2}\left(\begin{array}{c}
n+m-1-k \\
m-1
\end{array}\right)=2\left(\begin{array}{c}
n+m+1 \\
m+2
\end{array}\right)-\left(\begin{array}{c}
n+m \\
m+1
\end{array}\right)=f_{m+2,2}(n) \\
\sum_{k=1}^{n} k^{3}\left(\begin{array}{c}
n+m-1-k \\
m-1
\end{array}\right)=6\left(\begin{array}{c}
n+m+1 \\
m+3
\end{array}\right)+\left(\begin{array}{c}
n+m \\
m+1
\end{array}\right)=2 f_{m+3,3}(n)-f_{m+2,2}(n) .
\end{gathered}
$$

Proof. Formula (3.24) follows from (3.12) for $m-1$ instead of $m$.

Further, using repeatedly (3.24), the definition (2.1) and the identity $\left(\begin{array}{l}m-1 \\ m-1\end{array}\right)=\left(\begin{array}{l}m \\ m\end{array}\right)$ we obtain

$$
\begin{aligned}
\sum_{k=1}^{n} k\left(\begin{array}{c}
n+m-1-k \\
m-1
\end{array}\right) & =1\left(\begin{array}{c}
n+m-2 \\
m-1
\end{array}\right)+2\left(\begin{array}{c}
n+m-3 \\
m-1
\end{array}\right)+3\left(\begin{array}{c}
n+m-4 \\
m-1
\end{array}\right)+\cdots+n\left(\begin{array}{c}
m-1 \\
m-1
\end{array}\right) \\
& =\sum_{k=1}^{n}\left(\begin{array}{c}
n+m-1-k \\
m-1
\end{array}\right)+\sum_{k=2}^{n}\left(\begin{array}{c}
n+m-1-k \\
m-1
\end{array}\right)+\cdots+\sum_{k=n}^{n}\left(\begin{array}{c}
n+m-1-k \\
m-1
\end{array}\right) \\
& =\left(\begin{array}{c}
n+m-1 \\
m
\end{array}\right)+\left(\begin{array}{c}
n+m-2 \\
m
\end{array}\right)+\cdots+\left(\begin{array}{c}
m-1 \\
m-1
\end{array}\right)=\left(\begin{array}{c}
n+m \\
m+1
\end{array}\right)=f_{m+1,1}(n) .
\end{aligned}
$$

To prove (3.26) note that by Eqs. (3.24) and (3.25) and since $\left(\begin{array}{c}m-1 \\ m-1\end{array}\right)=\left(\begin{array}{c}m \\ m\end{array}\right)$ we have

$$
\begin{aligned}
\sum_{k=1}^{n} k^{2}\left(\begin{array}{c}
n+m-1-k \\
m-1
\end{array}\right) & =1^{2}\left(\begin{array}{c}
n+m-2 \\
m-1
\end{array}\right)+2^{2}\left(\begin{array}{c}
n+m-3 \\
m-1
\end{array}\right)+3^{2}\left(\begin{array}{c}
n+m-4 \\
m-1
\end{array}\right)+\cdots+n^{2}\left(\begin{array}{c}
m-1 \\
m-1
\end{array}\right) \\
& =1 \sum_{k=1}^{n}\left(\begin{array}{c}
n+m-1-k \\
m-1
\end{array}\right)+3 \sum_{k=2}^{n}\left(\begin{array}{c}
n+m-1-k \\
m-1
\end{array}\right)+5 \sum_{k=3}^{n}\left(\begin{array}{c}
n+m-1-k \\
m-1
\end{array}\right)+\cdots \\
& \cdots+(2 n-1) \sum_{k=n}^{n}\left(\begin{array}{c}
n+m-1-k \\
m-1
\end{array}\right) \\
& =1\left(\begin{array}{c}
n+m-1 \\
m
\end{array}\right)+3\left(\begin{array}{c}
n+m-2 \\
m
\end{array}\right)+5\left(\begin{array}{c}
n+m-3 \\
m
\end{array}\right)+\cdots+(2 n-1)\left(\begin{array}{c}
m \\
m
\end{array}\right) \\
& =\sum_{k=1}^{n}(2 k-1)\left(\begin{array}{c}
n+m-k \\
m
\end{array}\right)=2 \sum_{k=1}^{n} k\left(\begin{array}{c}
n+m-k \\
m
\end{array}\right)-\sum_{k=1}^{n}\left(\begin{array}{c}
n+m-k \\
m
\end{array}\right) \\
& =2\left(\begin{array}{c}
n+m+1 \\
m+2
\end{array}\right)-\left(\begin{array}{c}
n+m \\
m+1
\end{array}\right)=2 f_{m+2,1}(n)-f_{m+1,1}(n)=f_{m+2,2}(n),
\end{aligned}
$$

where the last equation follows from Lemma 3.10 for $k=2$. 
Finally, to prove (3.27) note that by Eqs. (3.24), (3.25) and (3.26) we have

$$
\begin{aligned}
\sum_{k=1}^{n} k^{3}\left(\begin{array}{c}
n+m-1-k \\
m-1
\end{array}\right) & =1^{3}\left(\begin{array}{c}
n+m-2 \\
m-1
\end{array}\right)+2^{3}\left(\begin{array}{c}
n+m-3 \\
m-1
\end{array}\right)+3^{3}\left(\begin{array}{c}
n+m-4 \\
m-1
\end{array}\right)+\cdots+n^{3}\left(\begin{array}{c}
m-1 \\
m-1
\end{array}\right) \\
& =1 \sum_{k=1}^{n}\left(\begin{array}{c}
n+m-1-k \\
m-1
\end{array}\right)+7 \sum_{k=2}^{n}\left(\begin{array}{c}
n+m-1-k \\
m-1
\end{array}\right)+19 \sum_{k=3}^{n}\left(\begin{array}{c}
n+m-1-k \\
m-1
\end{array}\right)+\cdots \\
& \cdots+\left(n^{3}-(n-1)^{3}\right) \sum_{k=n}^{n}\left(\begin{array}{c}
n+m-1-k \\
m-1
\end{array}\right) \\
& =1\left(\begin{array}{c}
n+m-1 \\
m
\end{array}\right)+7\left(\begin{array}{c}
n+m-2 \\
m
\end{array}\right)+19\left(\begin{array}{c}
n+m-3 \\
m
\end{array}\right)+\cdots+\left(3 n^{2}-3 n+1\right)\left(\begin{array}{c}
m \\
m
\end{array}\right) \\
& =\sum_{k=1}^{n}\left(3 k^{2}-3 k+1\right)\left(\begin{array}{c}
n+m-k \\
m
\end{array}\right) \\
& =3 \sum_{k=1}^{n} k^{2}\left(\begin{array}{c}
n+m-k \\
m
\end{array}\right)-3 \sum_{k=1}^{n} k\left(\begin{array}{c}
n+m-k \\
m
\end{array}\right)+\sum_{k=1}^{n}\left(\begin{array}{c}
n+m-k \\
m
\end{array}\right) \\
& =6\left(\begin{array}{c}
n+m+1 \\
m+3
\end{array}\right)-3\left(\begin{array}{c}
n+m+1 \\
m+2
\end{array}\right)-3\left(\begin{array}{c}
n+m+1 \\
m+2
\end{array}\right)+\left(\begin{array}{c}
n+m \\
m+1
\end{array}\right) \\
& =6 f_{m+3,1}(n)-6 f_{m+2,1}(n)+f_{m+1,1}(n) \\
& =2\left(3 f_{m+3,1}(n)-2 f_{m+2,1}(n)\right)-\left(2 f_{m+2,1}(n)-f_{m+1,1}(n)\right) \\
& =2 f_{m+3,3}(n)-f_{m+2,2}(n),
\end{aligned}
$$

where the last equation follows from Lemma 3.10 for $k=2$ and $k=3$ noting that $\left(\begin{array}{l}m-1 \\ m-1\end{array}\right)=\left(\begin{array}{c}m \\ m\end{array}\right)$.

Corollary 3.12 Let $\left(a_{n}\right)_{n \in \mathbb{N}}$ be an arithmetic progression with the initial value $a_{1}$ and the constant difference $d \in \mathbb{N}$, that is $a_{n}=a_{1}+(n-1) d$. Then for all $m \in \mathbb{N}\left(a_{n}^{(0)}:=a_{n}\right)$

$$
a_{n}^{(m)}=\sum_{k=1}^{n} a_{k}^{(m-1)}=\frac{m a_{1}+a_{n}}{m+1}\left(\begin{array}{c}
n+m-1 \\
m
\end{array}\right) .
$$

Proof. By Theorem 3.8 and applying Eqs. (3.24) and (3.25) it follows

$$
\begin{aligned}
a_{n}^{(m)} & =\sum_{k=1}^{n}\left(\begin{array}{c}
n+m-1-k \\
m-1
\end{array}\right)\left(a_{1}+(k-1) d\right) \\
& =a_{1} \sum_{k=1}^{n}\left(\begin{array}{c}
n+m-1-k \\
m-1
\end{array}\right)+d \sum_{k=1}^{n}\left(\begin{array}{c}
n+m-1-k \\
m-1
\end{array}\right)(k-1) \\
& =a_{1} f_{m, 1}(n)-d \cdot f_{m, 1}(n)+d \sum_{k=1}^{n} k\left(\begin{array}{c}
n+m-1-k \\
m-1
\end{array}\right) \\
& =\left(a_{1}-d\right) f_{m, 1}(n)+d \cdot f_{m+1,1}(n)=\left(\begin{array}{l}
a_{1}-d+d \cdot \frac{n+m}{m+1}
\end{array}\right) f_{m, 1}(n) \\
& =\frac{m a_{1}+a_{1}+(n-1) d}{m+1} f_{m, 1}(n)=\frac{m a_{1}+a_{n}}{m+1} f_{m, 1}(n)=\frac{m a_{1}+a_{n}}{m+1}\left(\begin{array}{c}
n+m-1 \\
m
\end{array}\right),
\end{aligned}
$$


since $f_{m+1,1}(n)=\left(\begin{array}{c}n+m \\ m+1\end{array}\right)=\frac{n+m}{m+1}\left(\begin{array}{c}n+m-1 \\ m\end{array}\right)=\frac{n+m}{m+1} f_{m, 1}(n)$.

Note that the special case $m=1$ gives the well-known formula $a_{n}^{(1)}=\frac{1}{2} n\left(a_{1}+a_{n}\right)$ for the sum of the first $n$ terms of an arithmetic progression.

We are now in a position to determine the fourth sequence $\left(s_{j}^{(0)}(n)\right)_{n \in \mathbb{N}}$ and all its hypersequences of the $m$ th generation $s_{j}^{(m)}(n):=\sum_{k=1}^{n} s_{j}^{(m-1)}(k), m \in \mathbb{N}$.

Proposition 3.13 For all $j, n \in \mathbb{N}$ :

$$
\begin{aligned}
s_{j}^{(0)}(n) & =\frac{1}{2} f_{1, j}(n)\left(2 f_{2, j}(n)-f_{1, j}(n)+1\right) \\
& =\frac{1}{2}(j n-(j-1))\left(j n^{2}-2(j-1) n+j\right)
\end{aligned}
$$

and for all $m \in \mathbb{N}_{0}$

$$
\begin{aligned}
s_{j}^{(m)}(n) & =j^{2} f_{m+3,3}(n)-\frac{j(4 j-3)}{2} f_{m+2,2}(n)+\frac{3 j^{2}-4 j+2}{2} f_{m+1,1}(n)-\frac{j(j-1)}{2} f_{m, 1}(n) \\
& =3 j^{2} f_{m+3,1}(n)-3 j(2 j-1) f_{m+2,1}(n)+\frac{7 j^{2}-7 j+2}{2} f_{m+1,1}(n)-\frac{j(j-1)}{2} f_{m, 1}(n) .
\end{aligned}
$$

Proof. By (3.4) and (2.2) we have

$$
\begin{aligned}
s_{j}^{(0)}(n) & =\sum_{k=1}^{f_{1, j}(n)} T_{j}(n, k)=\sum_{k=1}^{f_{1, j}(n)}\left(f_{2, j}(n)-f_{1, j}(n)+k\right) \\
& =f_{1, j}(n)\left(f_{2, j}(n)-f_{1, j}(n)\right)+\sum_{k=1}^{f_{1, j}(n)} k \\
& =f_{1, j}(n)\left(f_{2, j}(n)-f_{1, j}(n)\right)+\frac{1}{2} f_{1, j}(n)\left(f_{1, j}(n)+1\right) \\
& =\frac{1}{2} f_{1, j}(n)\left(2 f_{2, j}(n)-f_{1, j}(n)+1\right) .
\end{aligned}
$$

This proves the first expression in (3.29). The second expression can be obtained by substituting $f_{1, j}(n)=j n-(j-1)$ and $f_{2, j}(n)=\frac{1}{2} n(j n-(j-2))$ into this equation.

To prove the formula (3.30) we apply Theorem 3.8 to the sequence $\left(s_{j}^{(0)}(n)\right)_{n \in \mathbb{N}}$

$$
s_{j}^{(m)}(n)=\sum_{k=1}^{n}\left(\begin{array}{c}
n+m-1-k \\
m-1
\end{array}\right) s_{j}^{(0)}(k) .
$$

Expanding (3.29) we obtain

$$
s_{j}^{(0)}(n)=\frac{1}{2}(j n-(j-1))\left(j n^{2}-2(j-1) n+j\right)=\alpha_{j} n^{3}+\beta_{j} n^{2}+\gamma_{j} n+\delta_{j},
$$


where $\alpha_{j}=\frac{1}{2} j^{2}, \beta_{j}=-\frac{3}{2} j(j-1), \gamma_{j}=\frac{1}{2}\left(3 j^{2}-4 j+2\right)$ and $\delta_{j}=-\frac{1}{2} j(j-1)$. Substituting the last equation with these expressions into Eq.(3.31) we obtain

$$
s_{j}^{(0)}(n)=\alpha_{j} S_{3}(n)+\beta_{j} S_{2}(n)+\gamma_{j} S_{1}(n)+\delta_{j} S_{0}(n),
$$

where $S_{0}(n):=\sum_{k=1}^{n}\left(\begin{array}{c}n+m-1-k \\ m-1\end{array}\right), S_{1}(n):=\sum_{k=1}^{n} k\left(\begin{array}{c}n+m-1-k \\ m-1\end{array}\right)$,

$S_{2}(n):=\sum_{k=1}^{n} k^{2}\left(\begin{array}{c}n+m-1-k \\ m-1\end{array}\right)$, and $S_{3}(n):=\sum_{k=1}^{n} k^{3}\left(\begin{array}{c}n+m-1-k \\ m-1\end{array}\right)$. By Lemma 3.11 we have $S_{0}(n)=f_{m, 1}(n), S_{1}(n)=f_{m+1,1}(n), S_{2}(n)=f_{m+2,2}(n)$, and $S_{3}(n)=2 f_{m+3,3}(n)-$ $f_{m+2,2}(n)$. Hence,

$$
\begin{aligned}
s_{j}^{(m)}(n) & =\alpha_{j}\left(2 f_{m+3,3}(n)-f_{m+2,2}(n)\right)+\beta_{j} f_{m+2,2}(n)+\gamma_{j} f_{m+1,1}(n)+\delta_{j} f_{m, 1}(n) \\
& =2 \alpha_{j} f_{m+3,3}(n)+\left(\beta_{j}-\alpha_{j}\right) f_{m+2,2}(n)+\gamma_{j} f_{m+1,1}(n)+\delta_{j} f_{m, 1}(n) \\
& =j^{2} f_{m+3,3}(n)-\frac{j(4 j-3)}{2} f_{m+2,2}(n)+\frac{3 j^{2}-4 j+2}{2} f_{m+1,1}(n)-\frac{j(j-1)}{2} f_{m, 1}(n) .
\end{aligned}
$$

This is the first formula given in Eq.(3.30), the second is obtained by using Lemma 3.10 for $k=2$ and $k=3$ and this proves the proposition.

Remark 3.14 Note that formula (3.29) can also be derived by the fact that $s_{j}^{(0)}(n)$ is by definition the sum of the arithmetic progression with the initial value $T_{j}(n, 1)=c_{j}^{(0)}(n)=$ $f_{2, j}(n)-f_{1, j}(n)+1$, the constant difference $d=1$ and $f_{1, j}(n)=j n-(j-1)$ terms. The last term is then $f_{2, j}(n)$. Hence, by Corollary 3.12 for $m=1$, we have

$$
\begin{aligned}
s_{j}^{(0)}(n) & =\frac{1}{2} f_{1, j}(n)\left(f_{2, j}(n)-f_{1, j}(n)+1+f_{2,1}(n)\right) \\
& =\frac{1}{2}(j n-(j-1))\left(2 \cdot \frac{n}{2}(j n-(j-1))-(j n-(j-1))+1\right) \\
& =\frac{1}{2}(j n-(j-1))\left(j n^{2}-2(j-1) n+j\right)
\end{aligned}
$$

and this is formula (3.29).

The first few sequences $s_{j}^{(m)}(n)$ for $j, n \in \mathbb{N}$ and $m \in \mathbb{N}_{0}$ are:

- $m=0: s_{j}^{(0)}(n)=\frac{1}{2}(j n-(j-1))\left(j n^{2}-2(j-1) n+j\right)$.

Note that formula (3.30) for $m=0$ gives another representation of this sequence. Indeed, since $f_{3,1}(n)=\left(\begin{array}{c}n+2 \\ 3\end{array}\right), f_{2,1}(n)=\left(\begin{array}{c}n+1 \\ 2\end{array}\right), f_{1,1}(n)=n$ and $f_{0,1}(n)=1$, we obtain

$$
\begin{aligned}
s_{j}^{(0)}(n) & =3 j^{2} f_{3,1}(n)-3 j(2 j-1) f_{2,1}(n)+\frac{7 j^{2}-7 j+2}{2} f_{1,1}(n)-\frac{j(j-1)}{2} f_{0,1}(n) \\
& =3 j^{2} \frac{n}{6}(n+1)(n+2)-3 j(2 j-1) \frac{n}{2}(n+1)+\frac{7 j^{2}-7 j+2}{2} n-\frac{j(j-1)}{2} \\
& =\frac{1}{2} j^{2} n^{3}-\frac{3}{2} j(j-1) n^{2}+\frac{1}{2}\left(3 j^{2}-4 j+2\right) n-\frac{1}{2} j(j-1) .
\end{aligned}
$$


This cubic polynomial in $n$ has the simple root $\frac{j-1}{j}$ and therefore it can be factorized obtaining in this way formula (3.29).

Special cases are:

$j=1: s_{1}^{(0)}(n)=\frac{1}{2} n\left(n^{2}+1\right)$. These are the centered triangular pyramidal numbers $(\underline{\mathrm{A} 006003})$. It is also the row, column and diagonal magic sum in the magic square of order $n$, since $\frac{1}{n} \sum_{k=1}^{n^{2}}=\frac{1}{2} n\left(n^{2}+1\right)$.

$j=2: s_{2}^{(0)}(n)=(2 n-1)\left(n^{2}-n+1\right)=n^{3}+(n-1)^{3}$. These are the centered cube numbers (A005898).

$j=3: s_{3}^{(0)}(n)=\frac{1}{2}(3 n-2)\left(3 n^{2}-4 n+3\right)$. This sequence is not available in the OEIS [16].

- $m=1: s_{j}^{(1)}(n)=\frac{1}{8} n(j n-(j-2))\left(j n^{2}-(j-2) n+2\right)$.

This formula can be derived from (3.30) for $m=1$ noting that $f_{4,1}(n)=\left(\begin{array}{c}n+3 \\ 4\end{array}\right)$, $f_{3,1}(n)=\left(\begin{array}{c}n+2 \\ 3\end{array}\right), f_{2,1}(n)=\left(\begin{array}{c}n+1 \\ 2\end{array}\right)$ and $f_{1,1}(n)=n$. We obtain after some algebraic simplifications

$$
s_{j}^{(1)}(n)=\frac{1}{8} n\left(j^{2} n^{3}-2 j(j-2) n^{2}+\left(j^{2}-2 j+4\right) n-2(j-2)\right) .
$$

This cubic polynomial in $n$ has the simple root $\frac{j-2}{j}$ and therefore it can be factorized obtaining in this way the asserted formula.

Since $s_{j}^{(1)}(n)=f_{2,1}\left(f_{2, j}(n)\right)=f_{2,1}\left(\frac{1}{2}(j n-(j-1))\right)$ we see that $s_{j}^{(1)}(n)$ are (for all $j \in \mathbb{N}$ ) subsequences of the triangular numbers. Moreover, by Eq.(2.2)

$$
\begin{aligned}
s_{j}^{(1)}(n) & =T_{1}\left(f_{2, j}(n), f_{2, j}(n)\right)=\frac{1}{2} f_{2, j}(n)\left(f_{2, j}(n)-1\right)+f_{2, j}(n) \\
& =\frac{1}{2} f_{2, j}(n)\left(f_{2, j}(n)+1\right) .
\end{aligned}
$$

Special cases are:

$j=1: s_{1}^{(1)}(n)=\frac{1}{8} n(n+1)\left(n^{2}+n+2\right)$. These are the doubly triangularl numbers $(\underline{\mathrm{A} 002817})$. Since $\left.f_{2,1}\left(f_{2,1}(n)\right)=f_{2,1}\left(\frac{1}{2} n(n+1)\right)=\frac{1}{2}\left(\frac{1}{2} n(n+1)\right)\left(\frac{1}{2} n(n+1)\right)+1\right)=$ $\frac{1}{8} n(n+1)\left(n^{2}+n+2\right)$ this sequence is the second iterated function of $f_{2,1}(n)$.

$j=2: s_{2}^{(1)}(n)=\frac{1}{2} n^{2}\left(n^{2}+1\right)$ This is the sum of the first $n^{2}$ natural numbers ( $\underline{\mathrm{A} 037270})$.

$j=3: s_{3}^{(1)}(n)=\frac{1}{8} n(3 n-1)\left(3 n^{2}-n+2\right)$. This sequence is not available in the OEIS [16].

- $m=2: s_{j}^{(2)}(n)=\frac{1}{120} n(n+1)\left(3 j^{2} n^{3}-3 j(j-5) n^{2}-\left(2 j^{2}-5 j-40\right) n+2\left(j^{2}-10 j+20\right)\right)$. This formula can be derived from (3.30) for $m=2$ noting that $f_{5,1}(n)=\left(\begin{array}{c}n+4 \\ 5\end{array}\right)$, $f_{4,1}(n)=\left(\begin{array}{c}n+3 \\ 4\end{array}\right), f_{3,1}(n)=\left(\begin{array}{c}n+2 \\ 3\end{array}\right)$ and $f_{2,1}(n)=\left(\begin{array}{c}n+1 \\ 2\end{array}\right)$. We obtain after some lengthy algebraic simplifications the asserted formula. 
Special cases are:

$j=1: s_{1}^{(2)}(n)=\frac{1}{120} n(n+1)(n+2)\left(3 n^{2}+6 n+11\right)$. This sequence is not available in the OEIS [16].

$j=2: s_{2}^{(2)}(n)=\frac{1}{60} n(n+1)(2 n+1)\left(3 n^{2}+3 n+4\right)=\frac{\left((n+1)^{6}-n^{6}\right)-\left((n+1)^{2}-n^{2}\right)}{60}$.

These are the polynexus numbers of order 6 (A079547).

$j=3: s_{3}^{(2)}(n)=\frac{1}{120} n(n+1)\left(27 n^{3}+18 n^{2}+17 n-2\right)$. This sequence is not available in the OEIS [16].

\section{The Case $T_{1}(n, k)$}

Recall that by Theorem $2.2 T_{1}(n, k)=\frac{1}{2} n(n-1)+k, k \in[n]$ and that, conversely, by Theorem 2.4, the number $N$ is in the $n$th row and $k$ th column of the array $T_{1}(n, k)$, where $n=\left\lfloor\frac{1}{2}(1+\sqrt{8 N-7})\right\rfloor$ and $k=N-\frac{1}{2} n(n-1)$. Note that in this and in the next chapter we will use repeatedly the rules $\lceil-x\rceil=-\lfloor x\rfloor$ for all $x \in \mathbb{R}$ (cf. [7, Eq.(3.4), p. 68]) and $\lfloor x\rfloor+n=\lfloor x+n\rfloor$ (resp. $\lceil x\rceil+n=\lceil x+n\rceil$ for all $x \in \mathbb{R}$ and $n \in \mathbb{Z}$ (cf. [7, Eq.(3.6), p. 69]).

Remark 4.1 If $N \in \mathbb{N}$ is in the $n$th row of $T_{1}(n, k)$, where $n=\left\lfloor\frac{1}{2}(1+\sqrt{8 N-7})\right\rfloor$, then $N+1$ is also in the same row if and only if $N$ is not a triangular number, whereas $N+1$ is in the $(n+1)$ st row if and only if $N$ is a triangular number. This means that $\mid \frac{1}{2}(1+$ $\sqrt{8 N-7})\rfloor=\left\lfloor\frac{1}{2}(1+\sqrt{8(N+1)-7})\right\rfloor=\left\lfloor\frac{1}{2}(1+\sqrt{8 N+1})\right\rfloor \Leftrightarrow N$ is $\underline{\text { not a }}$ a triangular number and $\left\lfloor\frac{1}{2}(1+\sqrt{8(N+1)-7})\right\rfloor=\left\lfloor\frac{1}{2}(1+\sqrt{8 N+1})\right\rfloor=\left\lfloor\frac{1}{2}(1+\sqrt{8 N-7})\right\rfloor+1 \Leftrightarrow N$ is a triangular number. Both assertions can be expressed more concisely as follows:

$$
\left\lfloor\frac{1}{2}(1+\sqrt{8 N+1})\right\rfloor-\left\lfloor\frac{1}{2}(1+\sqrt{8 N-7})\right\rfloor=(N \text { is a triangular number })
$$

or, equivalently,

$$
\left\lfloor\frac{1}{2}(-1+\sqrt{8 N+1})\right\rfloor-\left\lfloor\frac{1}{2}(-1+\sqrt{8 N-7})\right\rfloor=(N \text { is a triangular number })
$$

Theorem 4.2 Let $\varphi(n):=\frac{1}{2}(2 n+1-\sqrt{8 n+1}), n \in \mathbb{N}$. Then for all $n \geq 2$ :

$$
T_{1}(n-1, n-1)=\left\lceil\varphi\left(T_{1}(n, n)\right)\right\rceil
$$

and for all $k \in \mathbb{N}$ and for all $n \geq k$ :

$$
T_{1}(n-1, k)=\left\lceil\varphi\left(T_{1}(n, k)\right)\right\rceil .
$$

Proof. Let $N$ be a triangular number, that is $N=\frac{1}{2} n(n+1), n \in \mathbb{N}$, then by Theorem 2.4 and by (4.1) $N$ is the $n_{1}(N)$ th row of $T_{1}(n, k)$, where

$$
\begin{aligned}
n_{1}(N) & =\left\lfloor\frac{1+\sqrt{8 N-7}}{2}\right\rfloor=\left\lfloor\frac{1+\sqrt{8 N+1}}{2}\right\rfloor-1=\left\lfloor\frac{1+\sqrt{4 n(n+1)+1}}{2}\right\rfloor-1 \\
& =\left\lfloor\frac{1+\sqrt{(2 n+1)^{2}}}{2}\right\rfloor-1=\frac{1+2 n+1}{2}-1=n
\end{aligned}
$$


and in the $k$ th column, where $k=N-\frac{1}{2} n(n-1)=\frac{1}{2} n(n+1)-\frac{1}{2} n(n-1)=n$. Hence, by Theorem 2.2, $N=T_{1}(n, n)$ and $\varphi\left(T_{1}(n, n)\right)=\varphi(N)=\frac{1}{2}(2 N+1-\sqrt{8 N+1})$. Substituting $N=\frac{1}{2} n(n+1)$ into the last equation and simplifying we obtain $\varphi\left(T_{1}(n, n)\right)=$ $\frac{1}{2} n(n-1)=T_{1}(n-1, n-1) \in \mathbb{N}$. Therefore, $\left\lceil\varphi\left(T_{1}(n, n)\right)\right\rceil=T_{1}(n-1, n-1)$ and this is Eq.(4.3).

Now let $N:=T_{1}(n, k)$ be a non-triangular number, i.e., for all $n \in \mathbb{N}: N=$ $T_{1}(n-1, k) \neq \frac{1}{2} n(n+1)$, then by Theorem $2.4, N$ is in the $n$th row and in the $k$ th column of $T_{1}(n, k)$, where $n=\left\lfloor\frac{1}{2}(1+\sqrt{8 N-7})\right\rfloor$ and $k=N-\frac{1}{2} n(n-1)$. Hence, by Remark 4.1 and using Eq.(4.1) we get

$$
\begin{aligned}
T_{1}(n-1, k) & =T_{1}(n, k)-(n-1)=N-n+1=N-\left\lfloor\frac{1+\sqrt{8 N-7}}{2}\right\rfloor+1 \\
& =N-\left\lfloor\frac{1+\sqrt{8 N+1}}{2}\right\rfloor+1=N-\left\lfloor\frac{-1+\sqrt{8 N+1}}{2}\right\rfloor \\
& =\left\lceil\frac{2 N+1-\sqrt{8 N+1}}{2}\right\rfloor=\lceil\varphi(N)\rceil=\left\lceil\varphi\left(T_{1}(n, k)\right)\right\rceil .
\end{aligned}
$$

The proof of the theorem is complete.

Remark 4.3 In the proof of Theorem 4.2 we have used the well-known property of the triangular numbers

$$
8 \cdot \frac{n(n+1)}{2}+1=(2 n+1)^{2}
$$

according to which $N$ is a triangular number if and only if $8 N+1$ is a perfect odd square number (cf. [4, p. 32]). Alternatively, for odd numbers $x: x^{2} \equiv 1(\bmod 8)$ or 8 is a divisor of $x^{2}-1$. This result was already known to Plutarch (about 100 A.D.).

Applying Theorem $4.2 n_{1}(N)-1$ times (noting that $N=T_{1}(n, k), n \in \mathbb{N}, k \in[n]$ ) we obtain

Corollary 4.4 Let $\varphi$ defined as in Theorem 4.2, $N \in \mathbb{N}$, and

$$
\psi(N):=\lceil\varphi(N)\rceil, \quad \ell(N):=n_{1}(N)-1=\left\lfloor\frac{-1+\sqrt{8 N-7}}{2}\right\rfloor .
$$

Then the $\ell$ th iterated function of $\psi$ is given by $\psi^{\ell(N)}(N)=1$, where $\psi^{1}(N):=\psi(N)$ and $\psi^{k+1}(N):=\psi\left(\psi^{k}(N)\right), k<\ell(N)$.

The meaning of Theorem 4.2 and Corollary 4.4 is as follows: Firstly, if $N \in \mathbb{N}$ is a triangular number, i.e. $N=T_{1}(n, n), n \in \mathbb{N}$, then $\psi(N)=\psi\left(T_{1}(n, n)\right)=\left\lceil\varphi\left(T_{1}(n, n)\right)\right\rceil$ is by 4.3 equal to the preceding triangular number $T_{1}(n-1, n-1)$ and secondly, if $N \in \mathbb{N}$ is not a triangular number, that is $N=T_{1}(n, k), n \in \mathbb{N}, k \in[n-1]$, then $\psi(N)=\psi\left(T_{1}(n, k)\right)=\left\lceil\varphi\left(T_{1}(n, k)\right)\right\rceil=T_{1}(n-1, k)$ is by 4.4 exactly the number above 
$T_{1}(n, k)$ in the same column $k$. Hence, starting with an arbitrary $N \in \mathbb{N}$ in Table 1 we go a row upward in the same column until we reach a triangular number (in case $N$ is a triangular number there is nothing to do). Then we continue going upward in Table 1 along the principal diagonal until we reach after $\ell(N)=n_{1}(N)-1$ steps the first row with the value 1 .

\section{Application to the Tower of Hanoi with Four Pegs}

In 1989 Hinz [8] presented an iterative algorithm for the minimal solution of the Tower of Hanoi with four pegs (also known as the Reve's puzzle) and called it the presumed minimal solution of the Tower of Hanoi with four pegs. Meanwhile, in 2014 T. Bousch [2] proved in a brilliant article that the Frame-Stewart conjecture is true for the Reve's puzzle. Since the number of moves of the iterative solution by Hinz is exactly the same as those given by the Frame-Stewart conjecture we can speak of an iterative minimal solution of the Reve's puzzle. (Other different iterative minimal solutions for the Reve's puzzle have been given by van de Liefvoort [12] and Lu [13].) The minimum number of moves is given as the solution of the recurrence relation

$$
\nu(0)=0, \quad \forall n \in \mathbb{N}: \quad \nu(n)=\min _{0 \leq f<n}\left\{2 \nu(f)+2^{n-f}-1\right\},
$$

where any $f \in[n]_{0}:=\{0,1,2, \ldots, n-1\}$ for which $\nu(n)=2 \nu(f)+2^{n-f}-1$ holds, is called a minimum partition number for $n$. In general, this number is not unique. In [8, Corollary, pp. 136-137] it is shown that for $\varphi$ as defined in Theorem 4.2

$$
f(n)=\lfloor\varphi(N)\rfloor
$$

is a minimum partition number for $n$.

In this chapter we shall show that $\lceil\varphi(N)\rceil$ is a minimum partition number for $n$, too. To this end we recall the following recurrence relation (of first order) for $\nu(n)$ (cf. [11, Lemma 4.5 for $p=4$, p. 151] and [10, p. 159 for $p=4]$ )

$$
\nu(0)=0, \quad \forall n \in \mathbb{N}_{0}: \quad \nu(n+1)=\nu(n)+2^{\left\lfloor\frac{1}{2}(-1+\sqrt{8 n+1})\right\rfloor}=\nu(n)+2^{n-\lceil\varphi(n)\rceil} .
$$

By summation one gets from (5.3) the formula (cf. [11, Theorem 4.6 for $p=4$, p. 152])

$$
\forall n \in \mathbb{N}_{0}: \quad \nu(n)=\sum_{k=0}^{n-1} 2^{\left\lfloor\frac{1}{2}(-1+\sqrt{8 k+1})\right\rfloor}=\sum_{k=0}^{n-1} 2^{k-\lceil\varphi(k)\rceil} .
$$

Alternatively, from Eq.(5.3) we obtain a second recurrence relation (of second-order) for $\nu(n)$.

Corollary 5.1 For all $n \in \mathbb{N}$ :

$$
\nu(n+1)= \begin{cases}3 \nu(n)-2 \nu(n-1), & \text { if } n \text { is a triangular number } \\ 2 \nu(n)-\nu(n-1), & \text { otherwise }\end{cases}
$$


or, equivalently,

$$
\nu(n+1)=2 \nu(n)-\nu(n-1)+(\nu(n)-\nu(n-1)) \cdot(n \text { is a triangular number })
$$

with the initial values $\nu(0)=0, \nu(1)=1$.

Proof. By Eq. $(5.3)$ it is $\nu(n+1)-\nu(n)=2^{\left\lfloor\frac{1}{2}(-1+\sqrt{8 n+1})\right\rfloor}$ and for $n-1$ instead of $n$ we have $\nu(n)-\nu(n-1)=2^{\left\lfloor\frac{1}{2}(-1+\sqrt{8 n-7})\right\rfloor}$. Hence, by subtraction $\nu(n+1)-\nu(n)-$ $(\nu(n)-\nu(n-1))=2^{\left\lfloor\frac{1}{2}(-1+\sqrt{8 n+1})\right\rfloor}-2^{\left\lfloor\frac{1}{2}(-1+\sqrt{8 n-7})\right\rfloor}$ or, equivalently, $\nu(n+1)=2 \nu(n)-$ $\nu(n-1)+2^{\left\lfloor\frac{1}{2}(-1+\sqrt{8 n+1})\right\rfloor}-2^{\left\lfloor\frac{1}{2}(-1+\sqrt{8 n-7})\right\rfloor}$. By Eq. (4.2) one sees that when $n$ is not a triangular number, the two powers of 2 in the last equation are equal. Therefore, $\nu(n+1)=2 \nu(n)-\nu(n-1)$. However, if $n$ is a triangular number, then again by Eq.(4.2) and noting that $2^{\left\lfloor\frac{1}{2}(-1+\sqrt{8 n-7})\right\rfloor}=\nu(n)-\nu(n-1)$ we obtain

$$
\begin{aligned}
\nu(n+1) & =2 \nu(n)-\nu(n-1)+2^{\left\lfloor\frac{1}{2}(-1+\sqrt{8 n-7})\right\rfloor} \\
& =2 \nu(n)-\nu(n-1)+(\nu(n)-\nu(n-1)) \\
& =3 \nu(n)-2 \nu(n-1)
\end{aligned}
$$

and this proves (5.5). The assertion (5.6) follows immediately from (5.5).

Finally, if $n$ is a triangular number, then by Eq.(5.5) for $n-1$ instead of $n$ we have $\nu(n)=2 \nu(n-1)-\nu(n-2)$ and therefore

$$
\begin{aligned}
\nu(n+1) & =2 \nu(n)+\nu(n)-2 \nu(n-1) \\
& =2 \nu(n)+2 \nu(n-1)-\nu(n-2)-2 \nu(n-1)=2 \nu(n)-\nu(n-2) .
\end{aligned}
$$

Hence, by Corollary 5.1 we obtain a third recurrence relation (of third-order) for $\nu(n)$.

Corollary 5.2 For all $n \geq 2$ :

$$
\nu(n+1)=2 \nu(n)- \begin{cases}\nu(n-2), & \text { if } n \text { is a triangular number }, \\ \nu(n-1), & \text { otherwise }\end{cases}
$$

or, equivalently,

$$
\nu(n+1)=2 \nu(n)-\nu(n-1)+(\nu(n-1)-\nu(n-2)) \cdot(n \text { is a triangular number })
$$

with the initial values $\nu(0)=0, \nu(1)=1, \nu(2)=3$.

The first few values of the sequence $(\nu(n))_{n \in \mathbb{N}_{0}}(\underline{\text { A007664 }})$ are

$(0,1,3,5,9,13,17,25,33,41,49,65,81,97,113,129,161,193,225,257,289,321,385, \ldots)$, whereas the first few values of the difference $(\nu(n+1)-\nu(n))_{n \in \mathbb{N}_{0}}(\underline{\text { A137688 }})$ are $(1,2,2,4,4,4,8,8,8,8,16,16,16,16,16,32,32,32,32,32,32,64,64,64,64,64,64,64, \ldots)$ (" $2^{n}$ appears $n+1$ times, $n \geq 0$ "). This is the non-decreasing sequence of numbers of the form $2^{j} \cdot 2^{k}, j, k \in \mathbb{N}_{0}$ (also denoted by $s^{(2)}:=\left(s_{i}^{(2)}\right)_{j \in \mathbb{N}}$ ). Note also that Eq.(5.4) can be written as $\nu(n)=\sum_{k=1}^{n} s_{i}^{(2)}$ (cf. [9, Corollary 5.6, p. 214]). 
Proposition 5.3 Let $\varphi$ be defined as in Theorem (4.2) and $\tilde{f}(n)=\lceil\varphi(N)\rceil$. Then

$$
\forall n \in \mathbb{N}: \quad \nu(n)=2 \nu(\tilde{f}(n))+2^{n-\tilde{f}(n)}-1,
$$

i.e., together with $\lfloor\varphi(N)\rfloor$ is $\lceil\varphi(N)\rceil$ a minimum partition number for $n$, too. In particular, these two values are coincident for a triangular number (cf. [5, Theorem 2, pp. 235-236] and [12, p. 91]).

Proof. Since $\tilde{f}(n)-f(n)=\lceil\varphi(n)\rceil-\lfloor\varphi(n)\rfloor=(n$ is not a triangular number $)$, we distinguish two cases:

1) $n$ is a triangular number, then $\tilde{f}(n)=f(n)$ and so Eq.(5.9) is established.

2) $n$ is not a triangular number, then $\tilde{f}(n)=f(n)+1$. Consider now the difference

$$
\Delta(n):=2 \nu(\tilde{f}(n))+2^{n-\tilde{f}(n)}-1-\nu(n)
$$

Inserting $\tilde{f}(n)=f(n)+1$ into the above equation and using Eqs.(5.2) and (5.3) for $f(n)$ instead of $n$ we obtain

$$
\begin{aligned}
\Delta(n) & =2 \nu(f(n)+1)+2^{n-f(n)-1}-\left(2 \nu(f(n))+2^{n-f(n)}-1\right) \\
& =2(\nu(f(n)+1)-\nu(f(n)))+2^{n-f(n)-1}-2^{n-f(n)} \\
& =2^{1+f(n)-\lceil\varphi(f(n))\rceil}-2^{n-f(n)-1} .
\end{aligned}
$$

The assertion (5.9) is proved if and only if the two exponents in the last equation are equal for all non-triangular numbers $n \in \mathbb{N}$. Hence, it remains to show that for all non-triangular numbers $N \in \mathbb{N}$ the following term

$$
\begin{aligned}
\delta(N): & =1+f(N)-\lceil\varphi(f(N))\rceil-(N-f(N)-1) \\
& =2(f(N)+1)-\lceil\varphi(f(N))\rceil-N \\
& =2\lceil\varphi(N)\rceil-\lceil\varphi(\lceil\varphi(N)\rceil-1)\rceil-N
\end{aligned}
$$

is exactly 0 . (Note that $\lceil\varphi(N)\rceil=f(N)+1$.)

In order to prove this, assume that $\lceil\varphi(N)\rceil$ is a triangular number. Then, by Theorem 4.2 there is a number $n \in \mathbb{N}$ such that $N=T_{1}(n+1, n)$ and by Eq.(4.4) $\lceil\varphi(N)\rceil=\left\lceil\varphi\left(T_{1}(n+1, n)\right)\right\rceil=T_{1}(n, n)$ and $\lceil\varphi(N)\rceil-1=T_{1}(n, n)-1=T_{1}(n, n-1)$. Hence, by Eq.(4.3) $\lceil\varphi(\lceil\varphi(N)\rceil-1)\rceil=\left\lceil\varphi\left(T_{1}(n, n-1)\right\rceil=T_{1}(n-1, n-1)\right.$. By Eq. $(2.5)$ for $n-1$ instead of $n$ and for $k=n-1$ and $j=1$ we obtain $\delta(N)=2 \cdot T_{1}(n, n)-T_{1}(n-$ $1, n-1)-T_{1}(n+1, n)=0$.

In the other case, if $[\varphi(N)]$ is not a triangular number, then by Theorem 4.2 there are $n \in \mathbb{N}$ and $k \in[n-1]$ such that $N=T_{1}(n, k),\lceil\varphi(N)\rceil=T_{1}(n-1, k)$. Hence, $\lceil\varphi(N)\rceil-1=T_{1}(n-1, k)-1=T_{1}(n-1, k-1)$ and $\lceil\varphi(\lceil\varphi(N)\rceil-1)\rceil=$ $\left\lceil\varphi\left(T_{1}(n-1, k-1)\right\rceil=T_{1}(n-2, k-1)\right.$. Therefore, by Eq.(2.5) for $n-2$ instead of $n$ and $k-1$ instead of $k$ and $j=1$ we have $\delta(N)=2 \cdot T_{1}(n-1, k)-T_{1}(n-2, k-1)-T_{1}(n, k)=0$.

Hence, for all $N \in \mathbb{N}$ we have obtained $\delta(N)=0$ and this proves the proposition. 
Note that Theorem 4.2 and Corollary 4.4 together with Proposition 5.3 tell us how many subtowers, that is regular states with $\underbrace{s s \ldots s}_{k} \underbrace{t t \ldots t}_{N-k}, s, t \in\{0,1,2,3\}, s \neq t, k \in \mathbb{N}$ and how many discs (this is the height of the subtower) have to be built in the iterative solution of the Tower of Hanoi with four pegs and $N$ discs by Hinz, namely $n=n_{1}(N)=\left\lfloor\frac{1}{2}(1+\sqrt{8 N-7})\right\rfloor$ subtowers of heights $1=\psi^{n-1}(N), \psi^{n-2}(N), \ldots, \psi^{1}(N)$ and $\psi^{0}(N)=N$, respectively. Note that the subtower of height $N$ is the ordinary tower of $N$ discs. (For more details on the Tower of Hanoi with more than three pegs we refer to [9, Chapter 5].) For example, let $N=25$, then by the above remark and looking at the red path in Figure 1 we obtain the sequence $25 \rightarrow 19 \rightarrow 14 \rightarrow 10 \rightarrow 6 \rightarrow 3 \rightarrow 1$, which tells us that in order to perform the iterative solution of the Reve's puzzle we have to build in all 7 subtowers oh heights 1,3,6,10,19 and 25, respectively.

However, this is only one of all possible ways to solve the Reve's puzzle, since for instance the sequence $25 \rightarrow 18 \rightarrow 13 \rightarrow 9 \rightarrow 5 \rightarrow 3 \rightarrow 1$ (see the blue path in Figure 1 ) is also a solution by Eq.(5.2). To conclude, we want to count the number of paths in Table 1 from $N$ to 1 , where the only allowed directions are north and northwest.

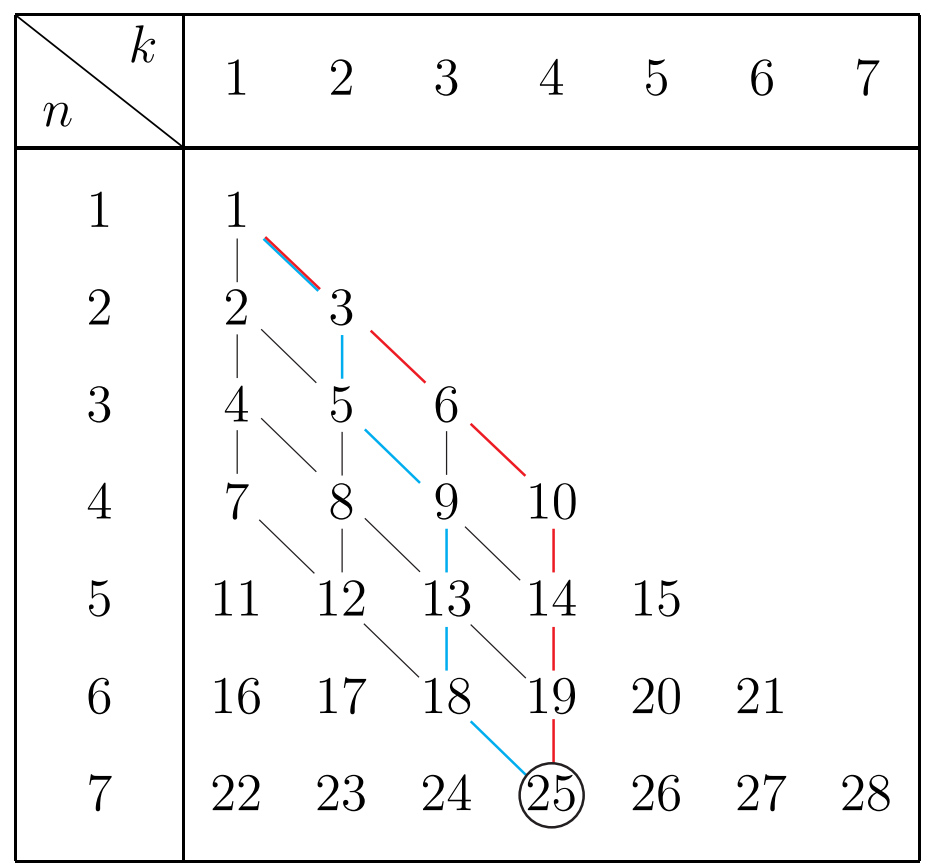

Figure 1: Two different optimal paths for the Reve's puzzle with 25 discs 
Corollary 5.4 Let $N \in \mathbb{N}$, then there are in Table 1 exactly

$$
\left(\begin{array}{c}
n_{1}(N)-1 \\
\left(\begin{array}{c}
n_{1}(N)+1 \\
2
\end{array}\right)-N
\end{array}\right)
$$

paths in north and northwest direction of length $\ell(N)=n_{1}(N)-1$ from $N \in \mathbb{N}$ to 1 , where $n_{1}(N)=\left\lfloor\frac{1}{2}(1+\sqrt{8 N-7})\right\rfloor$. The solution is unique only for all $N$ and $N+1$, where $N$ is a triangular number.

Proof. Let $N \in \mathbb{N}$, then by Theorem $2.4 N$ is in the $n$th row and $k$ th column of $T_{1}(n, k)$, where $n=n_{1}(N)=\left\lfloor\frac{1}{2}(1+\sqrt{8 N-7})\right\rfloor$ and $k=k_{1}(N)=N-\frac{1}{2} n_{1}(N) \cdot\left(n_{1}(N)-1\right)=$ $N-\left(\begin{array}{c}n_{1}(N) \\ 2\end{array}\right)$. The number of steps in north direction is given by $n_{1}(N)-k_{1}(N)=$ $n_{1}(N)-\left(N-\frac{1}{2} n_{1}(N) \cdot\left(n_{1}(N)-1\right)\right)=\left(\begin{array}{c}n_{1}(N)+1 \\ 2\end{array}\right)-N$. Since the total length of each path is $n_{1}(N)-1$, the number of steps in northwest direction is $n_{1}(N)-1-\left(n_{1}(N)-k_{1}(N)\right)=$ $k_{1}(N)-1$. A path can be described by a sequence of bits 0 and 1 of length $n_{1}(N)-1$, where the bit 0 indicates the north direction and 1 the northwest direction. Therefore, the total number of paths from $N$ to 1 is equal to the number of sequences of $n_{1}(N)-1$ bits of which $n_{1}(N)-k_{1}(N)=\left(\begin{array}{c}n_{1}(N)+1 \\ 2\end{array}\right)-N$ are 0 and $k_{1}(N)-1$ are 1 . This number is exactly the binomial coefficient given in Eq.(5.10).

If $N$ is a triangular number, then $k_{1}(N)=n_{1}(N)$ and hence $\left(\begin{array}{c}n_{1}(N)-1 \\ 0\end{array}\right)=1$. For $N+1, N$ a triangular number, we have $k_{1}(N)=1$, hence $\left(\begin{array}{l}n_{1}(N)-1 \\ n_{1}(N)-1\end{array}\right)=1$ and this proves the corollary.

Remark 5.5 Theorem 4.2, Corollary 4.4, Proposition 5.3 and Corollary 5.4 give a complete answer to the remark by H. E. Dudeney in [6, p. 132] (at least for the case with 4 pegs): "If the number of cheeses in the case of four stools is not triangular, ..., then there will be more than one way of making the piles and subsidiary tables will be required. This is the case with the Reve's 8 cheeses. But I will leave the reader to work out for himself the extension of the problem." Obviously, the words cheeses, stools and piles stand for discs, pegs and subtowers, respectively. Indeed, for $N=8$ we have $n=n_{1}(8)=\left\lfloor\frac{1}{2}(1+\sqrt{8 \cdot 8-7})\right\rfloor=4$ and by Eq.(5.10) $\left(\begin{array}{c}4-1 \\ \left(\begin{array}{c}4+1 \\ 2\end{array}\right)-8\end{array}\right)=\left(\begin{array}{l}3 \\ 2\end{array}\right)=3$ different sequences of subtowers have to be built, namely $8 \rightarrow 4 \rightarrow 2 \rightarrow 1,8 \rightarrow 5 \rightarrow 2 \rightarrow 1$ and $8 \rightarrow 5 \rightarrow 3 \rightarrow 1$ (cf. also [9, pp. 240-245], private communication by Hinz, 2019).

We remark that constructing all paths from a number $N$ up to the number 1 as defined in the above corollary one obtains the two-dimensional (complete) grid graph $P_{m} \square P_{n}$, that is the Cartesian product of the path graphs $P_{m}$ on $m=k_{1}(N)=N-\left(\begin{array}{c}n_{1}(N) \\ 2\end{array}\right)$ vertices and $P_{n}$ on $n=n_{1}(N)-k_{1}(N)+1=\left(\begin{array}{c}n_{1}(N)+1 \\ 2\end{array}\right)-N+1$ vertices (see Figure 2). Therefore $P_{m} \square P_{n}$ has $\left|P_{m} \square P_{n}\right|=\left|P_{m}\right| \cdot\left|P_{n}\right|=k_{1}(N) \cdot\left(n_{1}(N)-k_{1}(N)+1\right)$ vertices and ||$P_{m} \square P_{n}||=\left(\left|P_{m}\right|-1\right) \cdot\left|P_{n}\right|+\left(\left|P_{n}\right|-1\right) \cdot\left|P_{m}\right|=2\left|P_{m}\right| \cdot\left|P_{n}\right|-\left(\left|P_{m}\right|+\left|P_{n}\right|\right)=$ $2 k_{1}(N) \cdot\left(n_{1}(N)-k_{1}(N)+1\right)-\left(n_{1}(N)+1\right)$ edges. 


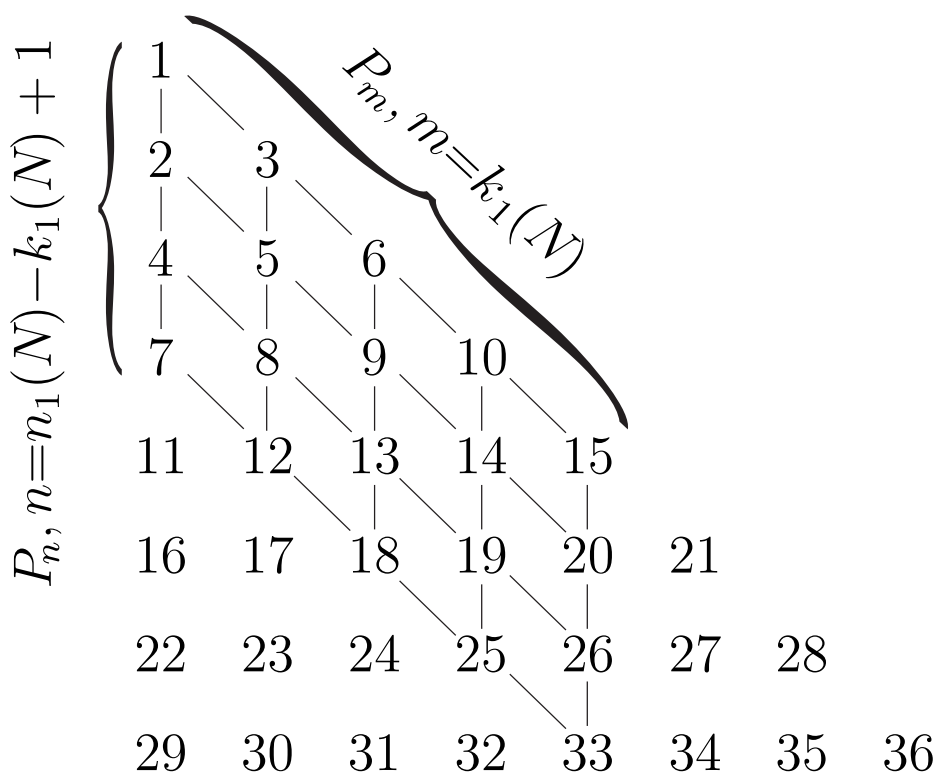

Figure 2: Grid graph $P_{m} \square P_{n}, m=5, n=4$

\section{Acknowledgments}

Thanks go to Andreas M. Hinz (Munich) for careful reading of the manuscript and for some valuable suggestions. I am also very grateful to Ciril Petr (Maribor) for technical support in generating Figure 1 and Figure 2 and to an anonymous referee for some useful remarks which helped to improve the paper.

\section{References}

[1] A. H. Beiler, Recreations in the Theory of Numbers, 2nd ed., Dover, New York, 1966.

[2] T. Bousch, La quatrième tour de Hanoï, Bull. Belg. Math. Soc. Simon Stevin 21 (2014) 895-912.

[3] L. Comtet, Advanced Combinatorics: The Art of Finite and Infinite Expansions, D. Reidel, Dordrecht, 1974.

[4] J. H. Conway, R. K. Guy, The Book of Numbers, Copernicus Springer New York, 1996.

[5] P. Cull and E. F. Ecklund, jr., On the Towers of Hanoi and Generalized Towers of Hanoi Problems, Congress. Numer. 35 (1982) 229-238. 
[6] H. E. Dudeney, The Canterbury puzzles and other curious problems, E. P. Dutton and Company, New York NY, 1908.

[7] R. L. Graham, D. E. Knuth, O. Patashnik, Concrete Mathematics, Addison-Wesley, Reading MA, 1989.

[8] A. M. Hinz, An Iterative Algorithm for the Tower of Hanoi with Four Pegs, Computing 42 (1989) 133-140.

[9] A. M. Hinz, S. Klavžar, C. Petr, The Tower of Hanoi - Myths and Maths, 2nd ed., Birkhäuser, Cham, 2018.

[10] S. Klavžar and U. Milutinović, Simple explicit formulas for the Frame-Stewart Numbers, Annals of Combinatorics 6 (2002) 157-167.

[11] S. Klavžar, U. Milutinović, C. Petr, On the Frame-Stewart algorithm for the multipeg Tower of Hanoi problem, Discrete Applied Mathematics 120 (2002) 141-157.

[12] A. van de Liefvoort, An iterative algorithm for the Reve's puzzle, The Computer Journal, Vol. 35, No. 1, (1992) 91-92.

[13] Xue-Miao Lu, An iterative solution for the 4-peg Towers of Hanoi, The Computer Journal, Vol. 32, No. 2, (1989) 187-189.

[14] I. Martinjak and I. Urbiha, A New Generalized Cassini Determinant, Colloquium Mathematicum, Vol. 145, No. 2, (2016) 209-218.

[15] I. Niven, Mathematics of Choice, MAA, Washington D.C., 1965.

[16] OEIS Foundation Inc. (2011), The On-Line Encyclopedia of Integer Sequences, https://oeis.org/ 
